\title{
Unbiased choice of global clustering parameters in single-molecule localization microscopy
}

\author{
Nold, Andreas ${ }^{1}$, Sun, Chao ${ }^{2}$, Heilemann, Mike ${ }^{3}$, Schuman, Erin M. ${ }^{2}$, and Tchumatchenko, \\ Tatjana *4,1 \\ ${ }^{1}$ Theory of Neural Dynamics, Max Planck Institute for Brain Research \\ ${ }^{2}$ Department of Synaptic Plasticity, Max Planck Institute for Brain Research \\ ${ }^{3}$ Institute for Physical and Theoretical Chemistry, Goethe-University Frankfurt \\ ${ }^{4}$ Institute of experimental epileptology and cognition research, University of Bonn
}

February 22, 2021

\begin{abstract}
Single-molecule localization microscopy resolves objects below the diffraction limit of light via sparse, stochastic detection of target molecules. Single molecules appear as clustered detection events after image reconstruction. However, identification of clusters of localizations is often complicated by the spatial proximity of target molecules and by background noise. Clustering results of existing algorithms often depend on user-generated training data or user-selected parameters, which can lead to unintentional clustering errors. Here we suggest an unbiased algorithm (FINDER) based on adaptive global parameter selection and demonstrate that the algorithm is robust to noise inclusion and target molecule density. We benchmark the most common density based clustering algorithms in test scenarios which we designed based on experimental datasets. We show that FINDER can keep the number of false positive inclusions low while also maintaining a low number of false negative detections in densely populated regions.
\end{abstract}

\section{Introduction}

Super-resolution microscopy has opened up new frontiers in biological and biomedical research by providing unprecedented molecular insights into the inner workings of cells [1, 2]. Classical light microscopy can only resolve structural features that are larger than the diffraction limit of light of a few hundred nanometers 3]. By overcoming the diffraction limit, super resolution microscopy has revealed long hidden mechanisms underlying intracellular transport processes [4] and the spatial organization of mRNA translation [5, 6]. The major feature of single-molecule localization microscopy (SMLM) is its ability to exploit the stochastic and sparse switching of fluorescence emission of specific labels binding to target molecules [7]. For example, in DNA-based point accumulation for imaging in nanoscale topography (DNA PAINT), each labelled target molecule is stochastically and repeatedly detected by transient binding of imager and docking strands [8]. Over time, each target molecule generates fluorescent detection events that cluster in space. Target molecules can be actin cytoskeleton [9], AMPA receptors [10 - or a well-defined DNA origami [11. However, SMLM

\footnotetext{
${ }^{*}$ Correspondence: tat@tchumatchenko.de
} 
data sets often contain localizations events that represent ambiguous information. For instance, a super resolved image may contain false positive localizations (i.e. background noise from nonspecific fluorescent signal that does not originate from a target molecule). Furthermore, in high density regions of target molecules, localizations from multiple target molecules can overlap or form complex structures [12].

Current state-of-the-art cluster detection algorithms rely on some form of prior, user-provided information. This information can be the type of localization patterns to be detected or prior experience. One of the most widely used [13, 14, 15, 16, 17, algorithms is the 'density-based spatial clustering algorithm' (DBSCAN) [18, 19]. It is intuitive, simple, fast for 2D datasets [20] - but the parameter choice suffers from human bias. To identify the 'most probable' clustering parameters based on prior knowledge, empirical methods for parameter identification have been proposed [19] (see Table S1). OPTICS [21] circumvent the use of a global clustering parameter by defining borders between clusters of localizations through changes in local point density. Fundamentally different approaches to clustering have also gained traction to circumvent the shortcomings of DBSCAN [22, 23, 24, 25]. In 2015, Rubin-Delanchy et al. have introduced a Bayesian parameter finding approach [26, 27] which starts from a user generated prior parameter set for a cluster-proposing algorithm, and subsequently computes a posterior probability for each parameter set. Also in 2015, Levet et al. introduced SR Tesselation, an algorithm which segments large-scale images into polygonal regions [28, 29] and is specialized to reveal spatial structures at multiple scales [30]. Most recently in 2020, Williamson et al. developed a machine-learning approach [31] that classifies localizations depending on their local neighborhood. This approach does not require the users to provide parameters. It also beats most classical algorithms in selected clustering challenges [31, but depends on training data sets.

Despite their successes, none of the current approaches have fully removed the dependency on prior knowledge - either a statistical model is needed, a reference density needs to be set, or a machine-learning model needs to be trained on a pre-selected data set. We address the problem of parameter sensitivity and user-generated bias by building on the widely applied DBSCAN [13, 32, 33, 34, 35, algorithm and propose an unbiased parameter selection which we call FINDER. FINDER minimizes dependencies on prior knowledge by leveraging what is usually seen as a distractor: false positive localizations, or 'noise'. The core principle of FINDER is to use information about the clustering variability with respect to the variation of the radial parameter, which is also described in [36], to then select the most robust clustering. Global noise levels therefore act as a lower boundary for the sensitivity of the algorithm, preventing over-segmentation and minimizing false positive cluster inclusions. To validate this approach, we use clusters which we identified in super-resolution images [8] to produce synthetic test sets, and compare the performance of FINDER with one of the currently best performing clustering algorithms, the adaptive machine-learning algorithm CAML [31. We also compare the performance to classical DBSCAN and density-based OPTICS clustering approaches 21. We show that our FINDER algorithm is independent of training data, it is computationally tractable while also showing a similar or better performance as measured by true positive detections, and reducing false-positive cluster detections and allowing for better interpretability.

\section{Results}

The assignment of clusters of localizations in super resolution microscopy is not trivial. Consider, for example, DNA PAINT data sets of super resolved neuronal AMPA receptor localizations. In Fig. 11 (left) one can see how difficult it is to identify clusters of localizations due to variable cluster sizes, high-noise and overlap. Similarly, data sets of DNA origami trimers (Fig. 1 right) indicate that identifying localizations that belong to single molecules of interest and separating these locations from the background noise is also a challenge. Algorithms can propose candidate clusters that correspond to single molecules of interest. However, verifying the reliability of the result is difficult because the ground truth is not always known 
and different parameter settings within the algorithm can lead to different outcomes. This means that the presence or absence of molecules of interest and their inferred location will vary depending on the parameter settings of the algorithm. In general, parameter settings, algorithm training and selection can become sources for clustering bias or errors.

To solve these challenges we propose the clustering algorithm, which we call FINDER and which we outline in Fig. 2 and detail in the method section "FINDER algorithm". To benchmark FINDER, we used super resolution images of structurally well-defined DNA origami trimers and tetramers. DNA origami are folded DNA nanostructures with well- defined binding sites for fluorophores [1]. As such, the ground truth of the geometry of binding sites is known: The algorithms should identify not more than 3 and 4 subclusters for each DNA origami trimer and tetramer, respectively. Note that the subclusters of the identified oligomers exhibit a considerable heterogeneity in their number of localizations per subcluster.

We start by comparing FINDER to the recently proposed machine-learning based clustering algorithms (CAML), which outperformed most classical clustering algorithms in selected clustering test cases 31. CAML feeds density variations of the neighborhood of each point into a trained classifier to identify clusters. Here, we use the pre-trained models 'CAML 07VEJJ' and 'CAML 87B144' from Ref. [31, which consider the first 100 and 1000 neighboring points, respectively. In contrast, FINDER identifies an optimal radial parameter based on a similarity measure that is computed across an interval of probable parameters (see Methods for details). FINDER uses a density-based approach which is based on the core points defined in DBSCAN and then optimizes this parameter based on the parameter phase space in the particular data set. Core points are points that have at least minPts neighbors within radius $\varepsilon$. In DBSCAN, these core points are used to initiate clusters. Instead, in FINDER, all non-core points are identified as noise localizations and iteratively removed. We refer to this more conservative definition of core points as 'noise-free DBSCAN'. Our tests suggest that this self-contained definition of core points is more robust to noise and leads to a lower number of false positive cluster detections (see Figs. S6 [S11). In the supplemental information we also compare the performance of FINDER with a version of FINDER using the classical density-based algorithm DBSCAN and with OPTICS, showing a higher false positive detection rate and a lower robustness, respectively.

In Fig. 3, we show that the FINDER algorithm accurately predicts the number of binding sites of the DNA origami oligomer, even though the density of localizations is highly heterogeneous and the final result applies one radial parameter to the full image. Notably, the adaptive CAML algorithms lead to a wide variety of detected subclusters of localizations. CAML 07VEJJ detects 3-mers most often, but fails in detecting 4-mers. In constrast, CAML 87B144 is more accurate in detecting 4-mers than 3-mers. One explanation for this discrepancy is that, on average, the detected 3-mers have 156 localizations per subcluster, but 4-mers have 285 localizations per subcluster. This could explain why CAML 07VEJJ, which considers only the first 100 neighboring points, performs poorly for the tetramer dataset, but it does not explain the performance of CAML 87B144. It also does not explain the segmentation failure of CAML 87B144 if no random noise is added (see Fig. S5). This results suggest that a version of CAML which considers the first 1000 points would need to be retrained for such 3-mer and 4-mer datasets. Retraining of the model is one possibility to include global information into local clustering decisions. However, the ground truth statistics regarding inter-and intra-cluster distance in experimentally recorded data sets is not a priori known. It is therefore hard to define a good training data set without introducing user-generated training biases. This highlights a challenge that adaptive algorithms share: information about the local neighborhood is used for clustering decisions - but often, these decisions need information about the global properties of the dataset - such as noise intensity or cluster separation. Often, these global properties are assumed a priori through user-defined parameters or training data. This potential source of bias is avoided by FINDER, which systematically probes the full dataset to identify one set of global parameters for an easily interpretable density-based clustering algorithm.

As a second benchmark, we employed two libraries of unit clusters of localizations: (1) manually identified 
a

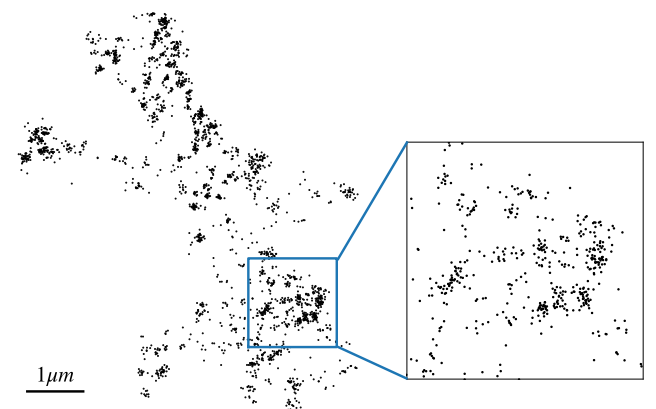

b

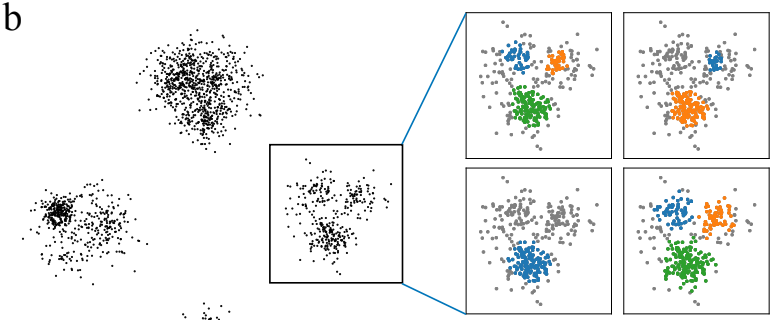

$\underline{100 \mathrm{~nm}}$

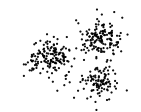

Figure 1: Single-molecule localization microscopy (SMLM) datasets exhibit density variations, noise inclusion and can lead to different cluster analysis results. a Localizations representing AMPA-receptors in a dendritic segment of a neuron [10]. b Example of four DNA origami trimers, with different clustering results for different parameters choices. The FINDER algorithm we propose here identifies parameters that lead to a statistically reliable assignment of clusters of localizations.
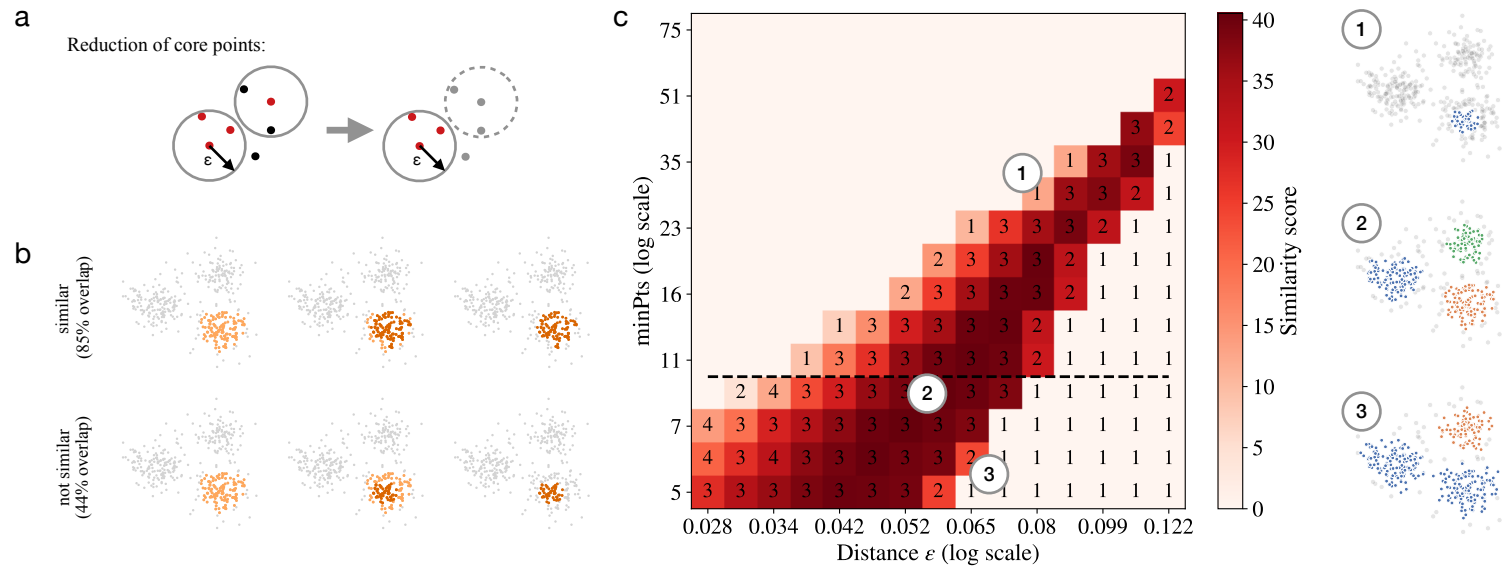

Figure 2: Schematics of FINDER algorithm a In DBSCAN, a new cluster is initiated when at least one core point (shown in red) is present that has at least minPts other points within distance $\varepsilon$ from the core point (see circles, left). Inspired by DBSCAN, the clustering algorithm used in FINDER iteratively removes non-core points (shown in black) which results in a more frequent identification of noise localizations (grey points, right). b Two clustering assigments are considered similar, if the number of matching localizations is greater than the number of unmatched localizations. Example of two similar cluster assignments (top row) and two non-similar cluster assignments (bottom row). c Phase space of possible clustering outcomes. FINDER defines a similarity score which is the number of similar clusters within the parameter space, for a given clustering. The dashed line represents minPts $=10$, which we used subsequently as a constant global parameter for FINDER (see Figs. S17, S18 for an analysis of the variations of minPts and phasespace plots of heterogeneous datasets). (1)-(3) represent three possible clustering outcomes within the parameter space. The parameters used for (1)-(3) correspond to the location of the respective number in the phase diagram, respectively. 


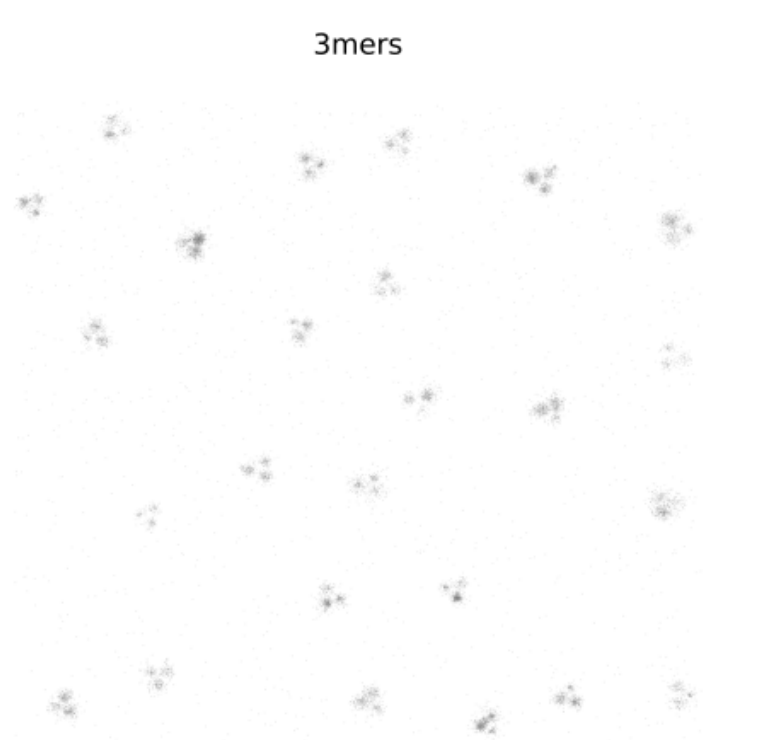

$500 \mathrm{~nm}$
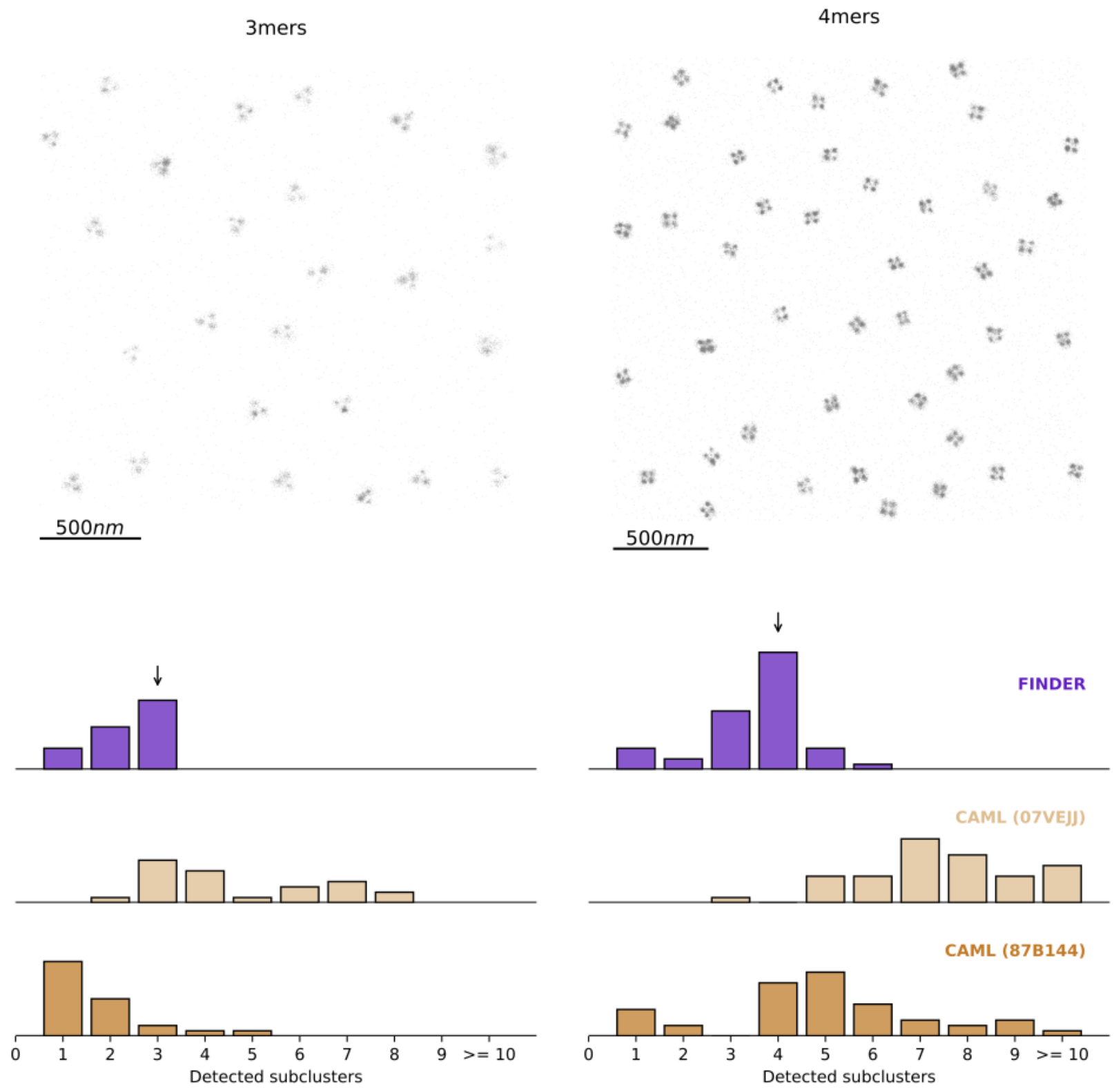

Figure 3: Performance of clustering algorithms for an image composed of 25 DNA-Origami 3-mers (left) and 44 4-mers (right), with added random noise localizations (10\% of cluster localizations). The optimal radial parameters identified by FINDER for DBSCAN (noisefree) are $\varepsilon=9.2 \mathrm{~nm}$ and $\varepsilon=4.7 \mathrm{~nm}$ for $\mathrm{minPts}=10$ for the trimer and the tetramer dataset, respectively. The histograms show the distribution of the number of subclusters detected for each 3-mer and 4-mer in our test data set, respectively. See Fig. S5 for clustering results without added noise, leading to a segmentation failure of CAML 87B144, suggesting that retraining is necessary. 
clusters in a SMLM dataset of a synapse [10, which contain on average only 17 localizations per cluster (see Fig. S1 a) and (2) manually identified sub-clusters of DNA-origami trimers, with on average 113 localizations per cluster (see Fig. S1 b). We re-arranged these unit clusters in three different configurations and added random noise points. These surrogate test data sets provide a ground-truth, while also retaining the biological variability of the cluster geometry. Our clustering outcomes are summarized in Fig 4 . As expected, the CAML 07VEJJ model fails for the set of unit clusters where the number of points per cluster can be larger than the number of points considered (100, see Fig. S1 b). Interestingly, in several test cases CAML 87B144, which considers the first 1000 neighboring points, also fails for the smaller set of unit clusters (see Fig. S1 a). If the 'correct' CAML model is chosen, performance is good, with a high number of true positive and low number of false negative detections. For all test cases we considered here, FINDER leads to a similar number of true positive cluster detections as the CAML model that performs better for the given configuration. FINDER consistently results in the lowest number of false positive cluster detections. See Figs. S6 S11 for more expansive tests. This suggests that FINDER is able to identify global parameters which give robust results with low false positive detection rates.

Finally, we applied FINDER and other clustering algorithms on SMLM datasets for which the ground truth is not known. In Fig. 5, we show the clustering results for single-molecule localization DNA-PAINT data of newly synthesized proteins after global homeostatic scaling in neuronal dendrites [6] (see Fig. S13 for an analogous analysis for a dataset of neuronal AMPA-receptors). Most clusters of localizations detected by FINDER and the two CAML algorithms have 50 or fewer localizations. CAML (07VEJJ) leads to an abrupt cutoff of cluster sizes at 100, suggesting that typical clusters can exceed that size, and therefore more than 100 neighbors need to be included in the analysis. The cluster size distribution obtained using CAML (87B144) leads to a tail in which clusters with more than 150 localizations are found, and $40 \%$ of all localizations are identified as noise. In comparison, FINDER identifies $27 \%$ of all localizations as noise localizations. FINDER therefore includes more large clusters, which usually represent molecular aggregations, than CAML (87B144). The distribution of cluster sizes for CAML (87B144) (see second bin) and visual inspection of the clusters suggests that there is an over-segmentation, which may require additional filtering. We conclude that the cluster-size distributions are overall robust with respect to the choice of the algorithm. We also note that clustering-results provided by FINDER have the advantage of being easily interpretable and reproducible: For example here, a cluster is defined if 10 core points are found within $80.4 \mathrm{~nm}$, and a noise localization is defined if it does not have 10 localizations within that radius.

\section{Discussion}

The identification of clustered localizations in single-molecule localization microscopy data is a crucial step for quantifying proteins within nano-clusters [23, 37]. A bottleneck for automated image analysis is the identification of appropriate clustering parameters, particularly if target molecules themselves are clustered or if noise levels are high. Clustering algorithms used in scientific studies of SMLM datasets therefore need to fulfill many - sometimes contradicting - requirements. They have to be fast, robust with respect to parameter choice, and offer results that are easily interpretable. Manual parameter tuning needs to be avoided, as detailed knowledge about the system may not be readily available and can introduce human bias. Because control experiments with known ground truth clusterings are not always available, false positive cluster inclusions need to be minimized in order to avoid over- or misinterpretations of the data.

DBSCAN is one of the most popular clustering algorithms for SMLM data [14, 15, 16, 17, 13, 32, 33, 34, 35. Part of its appeal is the fact that its density-based clustering rule is intuitive and it can offer close-tooptimal results if parameters are chosen carefully [22. It has two input parameters: a radial parameter $\varepsilon$ and the minimal number of points minPts within this radial parameter needed to initiate a cluster. However it 
Low density clusters

a High overlap

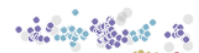

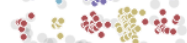

* \%

अ

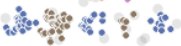

$200 \mathrm{~nm}$
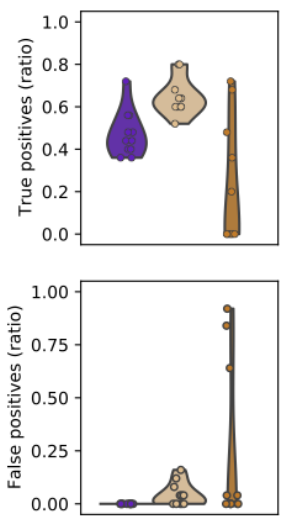

b High noise

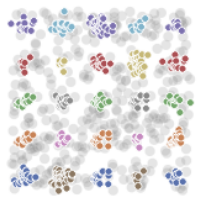

-
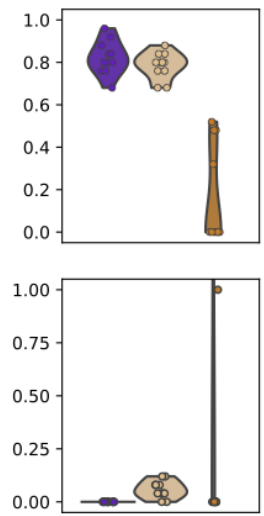

c Unstructured

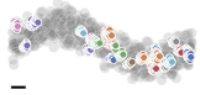

$-$
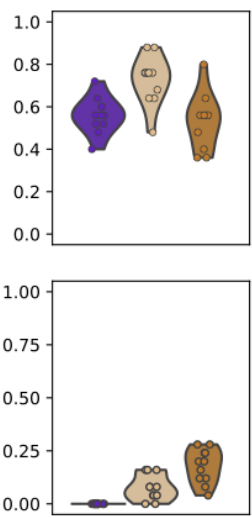

FINDER $\square$ CAML (07VEJI) d High overlap

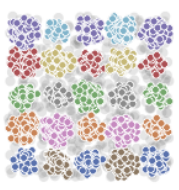

4. . .
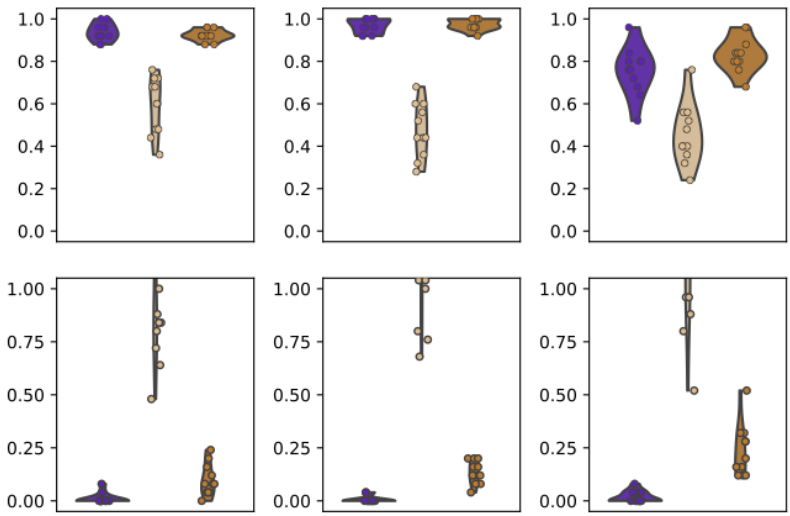

f Unstructured
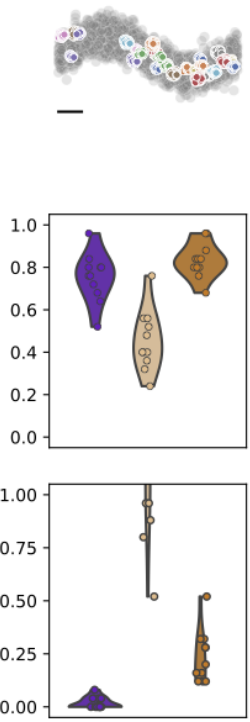

Figure 4: Performance of FINDER and CAML clustering algorithms across synthetic datasets which are composed of clusters of localizations from two libraries. Low-density clusters (a-c) are composed of clusters from a SMLM dataset of a a synapse [10] with an average of 17 localizations per cluster. Highdensity clusters (e-f) are composed of manually identified sub-clusters of DNA-origami trimers with on average 113 localizations per cluster. (a,d) High overlap: A grid of $5 \times 5$ clusters with distances equal to the maximal cluster diameter in the dataset, and with $20 \%$ random noise localizations (as a fraction of clustered localizations). (c,d) High noise: A grid of $5 \times 5$ clusters with distances equal to the 1.5 times the maximal cluster diameter in the dataset, is superimposed with an equal number $(100 \%)$ of random noise localizations. (e,f) Unstructured: 25 clusters are randomly distributed along a sinusoidal path, with 100\% and 150\% added random noise localizations (as a fraction of clustered localizations) in c and f, respectively. The top row shows one instance of a randomized pattern for each case, with highlighted ground truth clusters. For further detail, see supplemental figures: S6 S11, and Fig. S16. 

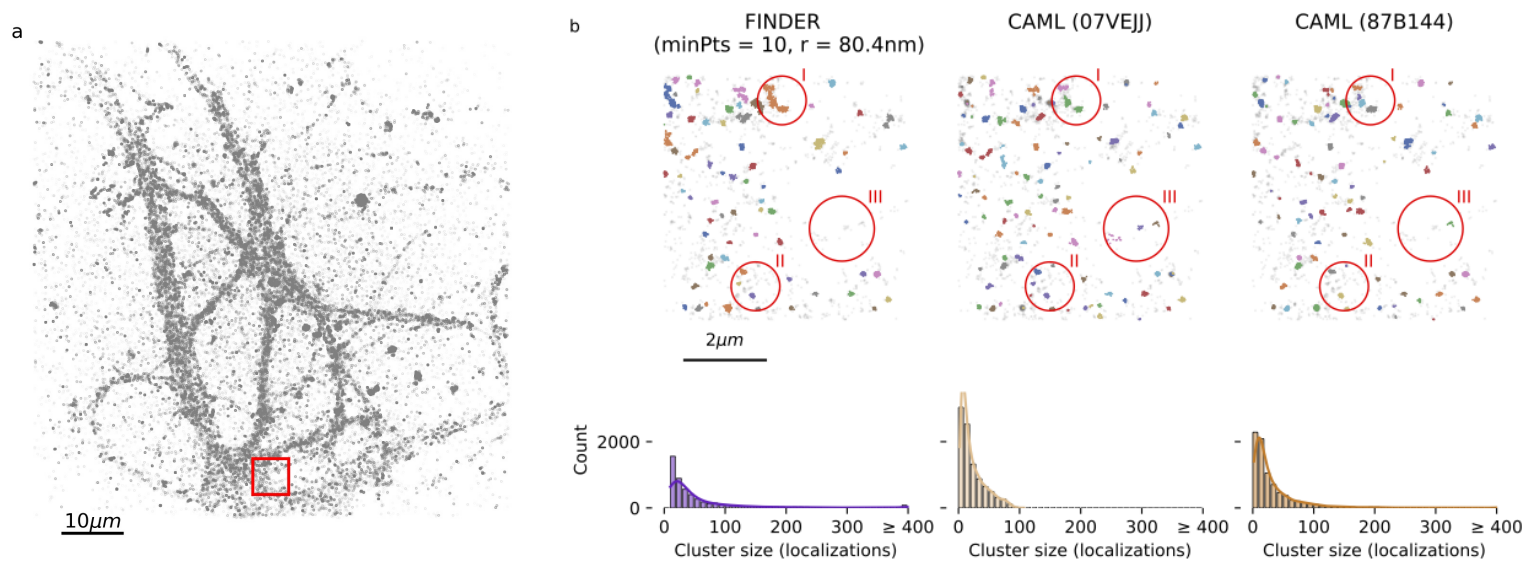

Figure 5: Analysis of newly synthesized proteins in neuronal dendrites in DNA-PAINT data [6]. Left: Localizations analyzed using FINDER, CAML (07VEJJ) and CAML (87B144) [31. Right: The top row depicts a section of the full field of view corresponding to the red rectangle in the left panel. Detected clusters are highlighted as colored points and localizations that were classified as noise are shown as grey points. The optimal parameters identified by FINDER for DBSCAN (noisefree) for minPts $=10$ are $\varepsilon=80.4 \mathrm{~nm}$. FINDER, CAML (07VEJJ) and CAML (87B144) assigned 18.9\%, $0 \%$ and $1.4 \%$ of all localizations to clusters with more than 400 localizations, respectively. See red circle (I) for an example of a large cluster. The overall structure of the results is similar (eg. red circle (II)), but FINDER sets a higher threshold for the selection of small clusters. Therefore, it identifies more clusters with a low number of localizations (cluster size $<25$ ) as noise, see eg. red circle (III). See Fig. S14 for the statistics showing the 10th-neighbor distances, Fig. S12 for an overview of the localizations not identified as noise, and for large clusters for each algorithm, and Fig. S17 for clustering outcomes within the full phasespace. See Fig. S13 for an analogous analysis of super resolved neuronal AMPA receptor localizations from [10].

is not always clear how to set the radial parameter manually for heterogeneous cluster sizes or datasets with changing density. Heuristic rules for setting these parameters exist [19, 22, but in many examples lead to ambiguity. For instance, the optimal value for the radial parameter $\varepsilon$ is commonly estimated from the k-thneighbor curves, which may be not well-defined and vary with $k 22$. Several adaptations and improvements of DBSCAN have been proposed to deal with density-variations, and the parameter estimation problem 38, 39. Reverse-nearest-neighbor approaches such as RECORD [40, IS-DBSCAN [4], ISB-DBSCAN [42] and RNN-DBSCAN [43] only require setting one parameter $k$, and can deal with density-variations within the dataset. In many applications, however, parameters are manually chosen (see Table S1) which may not lead to optimal results when analyzing many datasets with differing localization densities.

Here, we pursue an approach that does not locally adapt to varying densities, but which sets a global threshold for the required cluster density for scenarios in which the radial parameter cannot unambiguously be extracted from k-th-neighbor curves. The idea is to leverage the information given by overall noise levels to set a global threshold for cluster inclusions and therefore minimize the number of false positive cluster inclusions. To tackle the issue of parameter choice in this scenario, while retaining the advantages of density-based clustering methods such as DBSCAN, we presented an unbiased, automated parameter identification algorithm FINDER. FINDER combines the following benefits: First, it does not require prior knowledge about the structure of clustered localizations and by that it side-steps the need for a statistical model, the need to perform supervised training on annotated data sets, or the need of manual parameter 
choices that might encompass user-generated biases. Second, FINDER leads to easily interpretable results and automatically defines parameters which are then applied across the full dataset. Finally, FINDER can be applied to large, heterogeneous data sets with clustering results which are robust to noise and signal overlap (Figs. 2, 3, 4).

In the absence of a known ground truth- as is the case in most scientific exploratory data analysisclustering algorithms need to be transparent, easily interpretable, and minimize false positive detections to avoid misinterpretation of data. In local adaptive algorithms, local density changes are employed to detect clusters of localizations. But it is often unclear on what basis the threshold for the local density change ist set. In 2020, the local machine-learning based clustering method (CAML [31) has been proposed. If the correctly trained CAML algorithm is chosen, clustering results are generally very good, with high true positive and low false positive detections (see Fig. 4). However in some cases, these methods can lead to severe over-segmentation (Figs. 3, 4, 5) and in other cases to under-counting of clusters (Fig. 33). For exploratory data analysis with an unknown ground truth, this sensitivity is critical, because one does not know which datasets are outside of the validity regime of a given trained model.

This oversegmentation can be avoided if the global parameter choice is used to transfer information from the global to the local scale. For instance, information at the global scale is the overall amount of noise, or the general heterogeneity of the clusters. The local scale is the neighborhood of each localization. By avoiding pre-filtering of noise, and by performing both noise-filtering and clustering in one step, FINDER uses the global information to set the local radial parameter $\varepsilon$, which then defines a threshold for clustering. In brief, FINDER selects the radial parameter which leads to the most robust clustering. Let us mention that for our test scenarios we set the minPts parameter to 10 localizations. This is in contrast to minPts $=4$ recommended for all 2D datasets by Ester et al. [18. In particular, systematic phase space explorations revealed that minPts $=4$ lead to high sensitivity of the results to noise, over-segmentation, and as a result a large number of false positive cluster detections (see Fig. S17 and Fig. S18). We also note that a more stringent cluster point definition based on iteratively removing non-core points leads to more robust clustering results that the classical DBSCAN algorithm.

We systematically benchmarked FINDER against existing algorithms using two sets of experimentally recorded clusters of localizations. We found that - despite using the same parameters across the full dataset the cluster inclusion and exclusion criteria used in FINDER perform robustly compared to trained machinelearning models. Our tests on synthetic data sets in the high noise and the high overlap regimes showed that density variations due to noise can lead to over-segmentation in adaptive clustering algorithms if an incorrect algorithm is used (see Fig. 4). For example, for synthetic reconstructions, FINDER performed similar to the better of two pre-trained CAML models. FINDER was able to minimize the number of false positive clusters while maintaining a high ratio of true positive clusters. We also tested FINDER on a dataset of DNA-origami trimers and tetramers and showed that FINDER reduces the number of false positive cluster detections and at the same time retains many true positive detections - leading to an accurate prediction of the underlying molecular structure.

In conclusion, we showed that performing noise identification, parameter-choice and clustering in one single post-processing step, such as proposed in FINDER, provides a reliable and unbiased method for a spatial analysis of SMLM data sets. In most experimental settings, the ground truth is not known, and therefore minimizing the number of false positive cluster detections is important to avoid erroneous interpretations of experimental results. We showed here that an all-in-one cluster identification can help limit the effect of human biases, and can speed up the interpretation of single molecule microscopy datasets. 


\section{Methods}

\section{FINDER algorithm}

The FINDER algorithm identifies the hyperparameters for a cluster-proposing algorithm. Here, we employ two cluster proposing algorithms: DBSCAN, as well as a version of DBSCAN based on iterative removal of non-core points (see Methods-section on "Noise-free DBSCAN" for details). Both algorithms take two parameters, which are a fixed minimum number of neighboring points ( $\mathrm{minPts}$ ), and a typical distance $(\varepsilon)$. FINDER determines the radial parameter through the following steps:

1. Compute the distribution of the distance to the $k$ th-nearest neighbors. Here, we set $k=10$ (see discussion, and Fig. S17 and Fig. S18.

2. Define the interval in which the algorithm will search for the second parameter $\varepsilon$ as the interval between the 10 th to the 90 th percentile of the distribution of $k$ th-nearest neighbors.

3. For the input dataset, compute clustering results at $n$ logarithmically distributed points across the interval (here, $n=15$, see Fig. S19 for robustness), using the cluster-proposing algorithm.

4. Compute the similarity score for each clustering result, see Methods section "Similarity of clusterings and similarity score".

5. The parameters that correspond to the clustering with the largest similarity score is selected.

The procedure is illustrated in Figs. S3 and S4.

\section{Noise-free DBSCAN cluster definition}

DBSCAN initiates clusters using core points. Core points are points which have a at least minPts neighboring points within a distance $\varepsilon$. Here, we modified this classic DBSCAN cluster definition to make it more robust to noise. First, we iteratively remove all non-core points from the dataset of localizations $X$ such that only core points remain (see Fig. S2). Next, FINDER partitions the remaining core points into clusters. The algorithm is illustrated by the following pseudo-code:

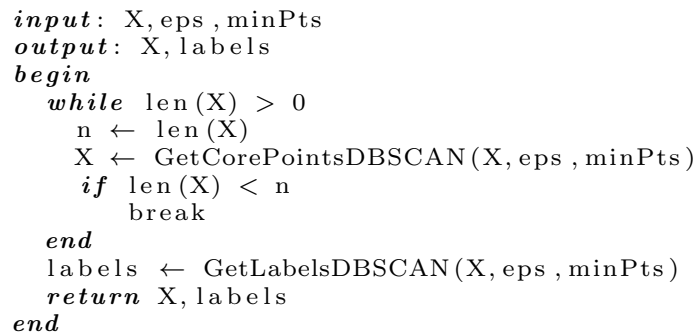

In all figures of the main manuscript, we used the noise-free DBSCAN cluster definition inside FINDER. For comparison with the classic DBSCAN cluster definition see Figs. S6 S11 in the supplementary material.

\section{Similarity of clusterings and the definition of the similarity score}

Let $\mathcal{C}_{i}=\left\{X_{1}, X_{2}, \ldots, X_{r}\right\}$ be a clustering of a set of points $\mathcal{P}=\left\{p_{1}, p_{2}, \ldots, p_{N}\right\}$. We define the similarity score of two clusterings $\mathcal{C}_{1}=\left\{X_{1}, X_{2}, \ldots, X_{r}\right\}$ and $\mathcal{C}_{2}=\left\{Y_{1}, Y_{2}, \ldots, Y_{t}\right\}$ as the sum of similar subsets within 
the partitions:

$$
S\left(\mathcal{C}_{1}, \mathcal{C}_{2}\right)=\sum_{i, j} s\left(X_{i}, Y_{j}\right)
$$

Two subsets $X_{i}$ and $Y_{j}$ are said to be similar if the number of overlapping points is larger than the number of non-overlapping points for each of the subsets:

$$
s\left(X_{i}, Y_{j}\right)=\left\{\begin{array}{ll}
1 & \text { if }\left|X_{i} \cap Y_{j}\right|>\max \left(\left|X_{i} \backslash\left(X_{i} \cap Y_{j}\right)\right|,\left|Y_{j} \backslash\left(X_{i} \cap Y_{j}\right)\right|\right. \\
0 & \text { otherwise }
\end{array} .\right.
$$

The similarity score of a clustering $\mathcal{C}_{i}$ within an assembly of clusterings $\mathcal{A}=\left\{\mathcal{C}_{1}, \mathcal{C}_{2}, \ldots, \mathcal{C}_{m}\right\}$ is defined as the sum of similarity scores:

$$
\bar{S}\left(\mathcal{C}_{i}, \mathcal{A}\right)=\sum_{j} S\left(\mathcal{C}_{i}, \mathcal{C}_{j}\right)
$$

\section{Generation of surrogate test data from DNA origami images}

To benchmark the performance of FINDER on experimental data sets and compare its performance to alternative existing clustering methods we used a DNA origami data set with a known cluster structure. We considered images of DNA origami containing three or four binding sites that we measured using DNAPAINT 8. Even though three or four localization clusters are expected for this DNA origami data set and this knowledge can provide a ground truth for clustering outcomes, we found that all clustering methods consistently detected a varying fraction of dimers. Upon visual inspection, we found that some expected trimers or tetramers appeared incomplete because some fluorophore binding sites were absent from some origami. We thus divided the trimer data into two groups: visibly resolved and visibly un-resolved trimers. We also introduced the group of resolved tetramers, in order to test the performance of all algorithms on a different geometric configuration See Fig. 3 for analysis of both groups.

In a second test, we accessed the robustness of the algorithms in the limit of overlapping clusters, in the high-noise limit, and with geometric constraints. We manually selected clusters representing single binding sites from resolved trimers (see Fig. $4 \mathrm{~b}$ ) and, in a second set, we manually selected from AMPA receptor images (see Fig. S1). We then re-assembled those monomers in pre-defined grid and path geometries, and with varying levels of added random noise localizations.

\section{Definition of true and false positive cluster detections}

In Fig. 4 a cluster $X$ from the ground truth clustering $\mathcal{C}_{1}$ is counted as being correctly detected by cluster $Y$ from clustering $\mathcal{C}_{2}$ if the overlap between both clusters covers at least $30 \%$ of the points of cluster $X$, i.e. if $|X \cap Y|>0.3|X|$ and if cluster $Y$ does not detect any other clusters of clustering $\mathcal{C}$, i.e. one cluster $Y$ cannot detect two clusters from $\mathcal{C}_{1}$. All clusters from $\mathcal{C}_{2}$ that can be attributed to a cluster of $\mathcal{C}_{1}$ in such a way are counted as true positives, and the remaining clusters of $\mathcal{C}_{2}$ count as false positives. In Fig. S16, we vary the overlap threshold between $0 \%$ and $100 \%$, and show robustness of the results with respect to this variation.

Note that other metrics for the similarity of two clusterings [43] such as the Adjusted Rand Index (ARI) [44] or the Normalized Mutual Information (NMI) [45], mix the similarity and the number of correctly identified clusters. In contrast, for the second benchmark in Fig. 4. we focus on how many clusters have been correctly and incorrectly identified. We therefore use a metric that uses a hard, binary, threshold for individual clusters. 


\section{Experimentally recorded super-resolution microscopy data DNA Origami data}

DNA origami containing 3 ('trimers', 3-fold symmetry, $55 \mathrm{~nm}$ interspacing) and 4 ('tetramers', 4 fold symmetry, $40 \mathrm{~nm}$ interspacing) binding sites (containing P1 docking oligos) were imaged on the same N-STORM system (Nikon, Japan) as the above reported AMPA-receptor data: an Eclipse Ti-E inverted microscope, equipped with a Perfect Focus System (Ti-PSF) and a motorized x-y stage. Total internal reflection fluorescence (TIRF) was adjusted using a motorized TIRF illuminator in combination with a $100 \mathrm{x}$ oil-immersion objective (CFL Apo TIRF, NA 1.49) with a final pixel size of $158 \mathrm{~nm}$. For imaging, $647 \mathrm{~nm}$ excitation wavelength was used, housed in a MLC400B (Agilent) laser combiner. An optical fiber guided the laser beam to the microscope body and via a dichroic mirror (T660LPXR, Chroma) to the sample plane. Fluorescence emission was separated from excitation light via a bandpass filter (ET705/72m, Chroma) and detected by an iXon Ultra EMCCD camera (DU - 897U-CS0-23 \#BV, Andor). The software NIS-Elements $\mathrm{Ar} / \mathrm{C}$ (Nikon) and $\mu$ Manager were used to control the setup and the camera. TIRF illumination was used for super-resolution acquisitions of DNA origami data with a power of 30-40 $\mathrm{mW}$, which was de- termined directly after the objective and under wide-field configuration. Time-lapse datasets with 24000 frames for DNA origami trimers and 10773 frames for DNA origami tetramers and 16 bit depth were acquired at 3.3 $\mathrm{Hz}$ frame rate and $5 \mathrm{MHz}$ camera read-out bandwidth; pre-amplification: 3; electron multiplying gain: 50. For DNA-PAINT imaging, the imaging buffer contains P1- Atto655 (CTAGATGTAT-Atto655, Eurofins Genomics) in $500 \mathrm{mM} \mathrm{NaCl}, \mathrm{pH}$ 7.3. The P1-Atto655 concentration was $10 \mathrm{nM}$ for origami trimer and tetramer data, and $0.5 \mathrm{nM}$ for AMPA receptor PAINT [10.

DNA-PAINT acquisitions were reconstructed using Picasso:Localize, a module of the Picasso software, by applying a minimal net gradient of 1500 . With Picasso:Render, drift corrections were applied based on the redundant cross-correlation (RCC), with a segmentation of 1000 was applied. Drift-corrected data was filtered using Picasso:Filter. To generate the DNA origami trimer and tetramer cluster datasets for cluster-identification validation, trimer and tetramer clusters were identified by eye using Picasso:Render and manually selected with a picking diameter of 2 camera pixels.

\section{Newly synthesized protein data}

Newly synthesized protein data was previously reported (see Ref. [6]). In brief, cultured neuron was incubated in a growth medium containing (Tetrodotoxin) TTX for $1 \mathrm{hr}$. 15 mins before the treatment ended, the neuron was metabolically labelled with AHA. The immuno-stained neuron samples were then imaged using DNAPAINT [8].

\section{AMPA-receptor data}

AMPA-receptor validation data was previously reported (see Ref. [10]). In brief, cultured neurons were stained by primary antibody against AMPA receptor GluA2 subunit before fixation and secondary antibody staining, in which the secondary antibody was modified to carry a P1 docking oligo. The immuno-stained neuron samples were then imaged using DNA-PAINT [8].

\section{Acknowledgements}

C.S. and A.N. acknowledge the support by the Add-on Fellowship for Interdisciplinary Life Science (Project number: 850027) of the Joachim-Herz Foundation. C.S. is supported by an EMBO long-term postdoctoral fellowship (EMBO ALTF 860-2018), HFSP Cross-Disciplinary Fellowship (LT000737/2019-C). M.H. is 
funded by the German Science Foundation (DFG, grant SFB 902) and the Bundesministerium für Bildung und Forschung (BMBF:eBio). EMS is funded by the Max Planck Society, DFG CRC 1080: Molecular and Cellular Mechanisms of Neural Homeostasis and DFG CRC 902: Molecular Principles of RNA-based Regulation and the European Research Council (ERC) under the European Union's Horizon 2020 research and innovation programme (grant agreement No 743216). T.T. is funded by the Max Planck Society and DFG CRC1080: Molecular and Cellular Mechanisms of Neural Homeostasis. We thank Ann-Christin Andres and Nina Deussner for contributing the tetramer and trimer origami data. A.N. thanks Carlos Wert-Carvajal for proof-reading and valuable feedback.

\section{References}

[1] Yaron M Sigal, Ruobo Zhou, and Xiaowei Zhuang. Visualizing and discovering cellular structures with super-resolution microscopy. Science, 361(6405):880-887, 2018.

[2] Lothar Schermelleh, Alexia Ferrand, Thomas Huser, Christian Eggeling, Markus Sauer, Oliver Biehlmaier, and Gregor PC Drummen. Super-resolution microscopy demystified. Nature cell biology, 21(1):72-84, 2019.

[3] Antoine G Godin, Brahim Lounis, and Laurent Cognet. Super-resolution microscopy approaches for live cell imaging. Biophysical journal, 107(8):1777-1784, 2014.

[4] Adish Dani, Bo Huang, Joseph Bergan, Catherine Dulac, and Xiaowei Zhuang. Superresolution imaging of chemical synapses in the brain. Neuron, 68(5):843-856, 2010.

[5] Anne-Sophie Hafner, Paul G Donlin-Asp, Beulah Leitch, Etienne Herzog, and Erin M Schuman. Local protein synthesis is a ubiquitous feature of neuronal pre-and postsynaptic compartments. Science, 364(6441):eaau3644, 2019.

[6] Chao Sun, Andreas Nold, Tatjana Tchumatchenko, Mike Heilemann, and Erin M. Schuman. The spatial scale of synaptic protein allocation during homeostatic plasticity. bioRxiv, (https://doi.org/10.1101/ 2020.04.29.068833), 2020.

[7] Markus Sauer and Mike Heilemann. Single-molecule localization microscopy in eukaryotes. Chemical reviews, 117(11):7478-7509, 2017.

[8] Joerg Schnitzbauer, Maximilian T Strauss, Thomas Schlichthaerle, Florian Schueder, and Ralf Jungmann. Super-resolution microscopy with DNA-PAINT. Nature protocols, 12(6):1198, 2017.

[9] Ke Xu, Hazen P Babcock, and Xiaowei Zhuang. Dual-objective storm reveals three-dimensional filament organization in the actin cytoskeleton. Nature methods, 9(2):185, 2012.

[10] Carolin Böger, Anne-Sophie Hafner, Thomas Schlichthärle, Maximilian T Strauss, Sebastian Malkusch, Ulrike Endesfelder, Ralf Jungmann, Erin M Schuman, and Mike Heilemann. Super-resolution imaging and estimation of protein copy numbers at single synapses with DNA-point accumulation for imaging in nanoscale topography. Neurophotonics, 6(3):035008, 2019.

[11] Christian Steinhauer, Ralf Jungmann, Thomas L Sobey, Friedrich C Simmel, and Philip Tinnefeld. DNA origami as a nanoscopic ruler for super-resolution microscopy. Angewandte Chemie International Edition, 48(47):8870-8873, 2009. 
[12] David Baddeley and Joerg Bewersdorf. Biological insight from super-resolution microscopy: what we can learn from localization-based images. Annual review of biochemistry, 87:965-989, 2018.

[13] Ulrike Endesfelder, Kieran Finan, Seamus J Holden, Peter R Cook, Achillefs N Kapanidis, and Mike Heilemann. Multiscale spatial organization of RNA polymerase in escherichia coli. Biophysical journal, 105(1):172-181, 2013.

[14] Laura Tarancón Díez, Claudia Bönsch, Sebastian Malkusch, Zinnia Truan, Mihaela Munteanu, Mike Heilemann, Oliver Hartley, Ulrike Endesfelder, and Alexandre Fürstenberg. Coordinate-based colocalization-mediated analysis of arrestin clustering upon stimulation of the $\mathrm{C}-\mathrm{C}$ chemokine receptor 5 with RANTES/CCL5 analogues. Histochemistry and cell biology, 142(1):69-77, 2014.

[15] Philip R Nicovich, Dylan M Owen, and Katharina Gaus. Turning single-molecule localization microscopy into a quantitative bioanalytical tool. Nature protocols, 12(3):453, 2017.

[16] Marie-Lena IE Harwardt, Mark S Schröder, Yunqing Li, Sebastian Malkusch, Petra Freund, Shashi Gupta, Nebojsa Janjic, Sebastian Strauss, Ralf Jungmann, Marina S Dietz, et al. Single-molecule super-resolution microscopy reveals heteromeric complexes of MET and EGFR upon ligand activation. International Journal of Molecular Sciences, 21(8):2803, 2020.

[17] Sebastian Malkusch and Mike Heilemann. Extracting quantitative information from single-molecule super-resolution imaging data with LAMA-localization microscopy analyzer. Scientific reports, 6(1):14, 2016.

[18] Martin Ester, Hans-Peter Kriegel, Jörg Sander, Xiaowei Xu, et al. A density-based algorithm for discovering clusters in large spatial databases with noise. $K d d, 96(34): 226-231,1996$.

[19] Erich Schubert, Jörg Sander, Martin Ester, Hans Peter Kriegel, and Xiaowei Xu. DBSCAN revisited, revisited: why and how you should (still) use DBSCAN. ACM Transactions on Database Systems (TODS), 42(3):1-21, 2017.

[20] Junhao Gan and Yufei Tao. DBSCAN revisited: mis-claim, un-fixability, and approximation. In Proceedings of the 2015 ACM SIGMOD international conference on management of data, pages 519-530, 2015.

[21] Mihael Ankerst, Markus M Breunig, Hans-Peter Kriegel, and Jörg Sander. OPTICS: ordering points to identify the clustering structure. ACM Sigmod record, 28(2):49-60, 1999.

[22] A Mazouchi and JN Milstein. Fast optimized cluster algorithm for localizations (focal): a spatial cluster analysis for super-resolved microscopy. Bioinformatics, 32(5):747-754, 2016.

[23] Ismail M Khater, Ivan Robert Nabi, and Ghassan Hamarneh. A review of super-resolution singlemolecule localization microscopy cluster analysis and quantification methods. Patterns, 1(3):100038, 2020 .

[24] Mehrsa Pourya, Shayan Aziznejad, Michael Unser, and Daniel Sage. Graphic: Graph-based hierarchical clustering for single-molecule localization microscopy. bioRxiv, (https://doi.org/10.1101/2020.12. 22.423931 ), 2020.

[25] Jeremy A Pike, Abdullah O Khan, Chiara Pallini, Steven G Thomas, Markus Mund, Jonas Ries, Natalie S Poulter, and Iain B Styles. Topological data analysis quantifies biological nano-structure from single molecule localization microscopy. Bioinformatics, 36(5):1614-1621, 2020. 
[26] Patrick Rubin-Delanchy, Garth L Burn, Juliette Griffié, David J Williamson, Nicholas A Heard, Andrew P Cope, and Dylan M Owen. Bayesian cluster identification in single-molecule localization microscopy data. Nature methods, 12(11):1072-1076, 2015.

[27] Juliette Griffié, Michael Shannon, Claire L Bromley, Lies Boelen, Garth L Burn, David J Williamson, Nicholas A Heard, Andrew P Cope, Dylan M Owen, and Patrick Rubin-Delanchy. A Bayesian cluster analysis method for single-molecule localization microscopy data. Nature Protocols, 11(12):2499, 2016.

[28] Florian Levet, Eric Hosy, Adel Kechkar, Corey Butler, Anne Beghin, Daniel Choquet, and Jean-Baptiste Sibarita. SR-Tesseler: a method to segment and quantify localization-based super-resolution microscopy data. Nature methods, 12(11):1065, 2015.

[29] Florian Levet, Guillaume Julien, Rémi Galland, Corey Butler, Anne Beghin, Anaël Chazeau, Philipp Hoess, Jonas Ries, Grégory Giannone, and Jean-Baptiste Sibarita. A tessellation-based colocalization analysis approach for single-molecule localization microscopy. Nature communications, 10(1):1-12, 2019.

[30] David Baddeley. Detecting nano-scale protein clustering. Nature methods, 12(11):1019, 2015.

[31] David J Williamson, Garth L Burn, Sabrina Simoncelli, Juliette Griffié, Ruby Peters, Daniel M Davis, and Dylan M Owen. Machine learning for cluster analysis of localization microscopy data. Nature communications, 11(1):1-10, 2020.

[32] David Virant, Bjoern Traenkle, Julia Maier, Philipp D Kaiser, Mona Bodenhöfer, Christian Schmees, Ilijana Vojnovic, Borbála Pisak-Lukáts, Ulrike Endesfelder, and Ulrich Rothbauer. A peptide tag-specific nanobody enables high-quality labeling for dstorm imaging. Nature communications, 9(1):1-14, 2018.

[33] Cecilia P Sanchez, Christos Karathanasis, Rodrigo Sanchez, Marek Cyrklaff, Julia Jäger, Bernd Buchholz, Ulrich S Schwarz, Mike Heilemann, and Michael Lanzer. Single-molecule imaging and quantification of the immune-variant adhesin VAR2CSA on knobs of plasmodium falciparum-infected erythrocytes. Communications biology, 2(1):1-9, 2019.

[34] Amulya Nidhi Shrivastava, Virginie Redeker, Laura Pieri, Luc Bousset, Marianne Renner, Karine Madiona, Caroline Mailhes-Hamon, Audrey Coens, Luc Buée, Philippe Hantraye, et al. Clustering of tau fibrils impairs the synaptic composition of $\alpha 3-\mathrm{Na}+/ \mathrm{K}+-\mathrm{ATP}$ ase and AMPA receptors. The EMBO journal, 38(3):e99871, 2019.

[35] Amulya Nidhi Shrivastava, Luc Bousset, Marianne Renner, Virginie Redeker, Jimmy Savistchenko, Antoine Triller, and Ronald Melki. Differential membrane binding and seeding of distinct $\alpha$-synuclein fibrillar polymorphs. Biophysical Journal, 2020.

[36] Mattia Marenda, Elena Lazarova, Sebastian van de Linde, Nick Gilbert, and Davide Michieletto. Superstructure: a parameter-free super-structure identifier for smlm data. bioRxiv, 2020.

[37] Jack W Shepherd and Mark C Leake. Localization microscopy: a review of the progress in methods and applications. arXiv preprint arXiv:2011.03296, 2020.

[38] Tariq Ali, Sohail Asghar, and Naseer Ahmed Sajid. Critical analysis of dbscan variations. In 2010 International Conference on Information and Emerging Technologies, pages 1-6. IEEE, 2010.

[39] Kamran Khan, Saif Ur Rehman, Kamran Aziz, Simon Fong, and Sababady Sarasvady. DBSCAN: Past, present and future. In The fifth international conference on the applications of digital information and web technologies (ICADIWT 2014), pages 232-238. IEEE, 2014. 
[40] Soujanya Vadapalli, Satyanarayana R Valluri, and Kamalakar Karlapalem. A simple yet effective data clustering algorithm. In Sixth International Conference on Data Mining (ICDM'06), pages 1108-1112. IEEE, 2006.

[41] Carmelo Cassisi, Alfredo Ferro, Rosalba Giugno, Giuseppe Pigola, and Alfredo Pulvirenti. Enhancing density-based clustering: Parameter reduction and outlier detection. Information Systems, 38(3):317$330,2013$.

[42] Yinghua Lv, Tinghuai Ma, Meili Tang, Jie Cao, Yuan Tian, Abdullah Al-Dhelaan, and Mznah AlRodhaan. An efficient and scalable density-based clustering algorithm for datasets with complex structures. Neurocomputing, 171:9-22, 2016.

[43] Avory Bryant and Krzysztof Cios. RNN-DBSCAN: A density-based clustering algorithm using reverse nearest neighbor density estimates. IEEE Transactions on Knowledge and Data Engineering, 30(6):1109-1121, 2017.

[44] Lawrence Hubert and Phipps Arabie. Comparing partitions. Journal of classification, 2(1):193-218, 1985.

[45] Alexander Strehl and Joydeep Ghosh. Cluster ensembles - a knowledge reuse framework for combining multiple partitions. Journal of machine learning research, 3:583-617, 2002.

[46] Sandra Mayr, Fabian Hauser, Sujitha Puthukodan, Markus Axmann, Janett Göhring, and Jaroslaw Jacak. Statistical analysis of 3d localisation microscopy images for quantification of membrane protein distributions in a platelet clot model. PLoS computational biology, 16(6):e1007902, 2020. 


\section{Supplemental Information}

\begin{tabular}{|c|c|c|c|c|c|}
\hline Publication & SMLM method & Target molecule & $\operatorname{minPts}$ & $\mathrm{r}$ & Rationale \\
\hline $\begin{array}{l}\text { Endesfelder et al. [13] } \\
(2013)\end{array}$ & PALM & $\begin{array}{ll}\text { RNA } & \text { Poly- } \\
\text { merase } & \end{array}$ & 4 & $30 \mathrm{~nm}$ & $\begin{array}{l}\text { minPts as recom- } \\
\text { mended in [18] }\end{array}$ \\
\hline $\begin{array}{l}\text { Virant et al. } 32 \\
(2018)\end{array}$ & dSTORM & various targets & 6 & $40 \mathrm{~nm}$ & refer to Ref. [13] \\
\hline $\begin{array}{l}\text { Sanchez et al. } 33 \\
(2019)\end{array}$ & PALM & $\begin{array}{l}\text { VAR2CSA pro- } \\
\text { tein }\end{array}$ & 5 & $30 \mathrm{~nm}$ & - \\
\hline $\begin{array}{l}\text { Shrivastava et } \quad \text { al. } \\
(2019) \text { 34 }\end{array}$ & STORM & $\begin{array}{l}\text { Exogenous fib- } \\
\text { rillar Tau }\end{array}$ & 20 & $20 \mathrm{~nm}$ & - \\
\hline $\begin{array}{l}\text { Shrivastava et } \\
(2020) \text { 35] }\end{array}$ & STORM & $\begin{array}{l}\text { fibrillar a-Syn } \\
\text { polymorphs }\end{array}$ & 20 & $20 \mathrm{~nm}$ & - \\
\hline Mayr et al. (2020) 46] & 3D dSTORM & $\begin{array}{l}\text { CD } 41 \& C D 62 p \\
\text { proteins }\end{array}$ & 3 & $2 \times$ davg & $\begin{array}{l}\text { Tuned to filter } \\
\text { noise }\end{array}$ \\
\hline Mayr et al. (2020) 46] & 3D dSTORM & $\begin{array}{l}\text { CD } 41 \& C D 62 p \\
\text { proteins }\end{array}$ & 5 & $1.5 \times$ davg & $\begin{array}{l}\text { Tuned for cluster } \\
\text { assignment }\end{array}$ \\
\hline $\begin{array}{l}\text { Harwardt et al. (2020) } \\
{[16]}\end{array}$ & DNA-PAINT & $\begin{array}{l}\text { MET and epi- } \\
\text { dermal growth } \\
\text { factor receptor } \\
(\mathrm{EGFR})\end{array}$ & 10,15 & $10-15 \mathrm{~nm}$ & $\begin{array}{lr}\text { nearest } & \text { neighbor- } \\
\text { based } & \text { analysis } \\
(\mathrm{NeNA}) & \end{array}$ \\
\hline
\end{tabular}

Table S1: Overview of hyperparameters used for DBSCAN for the analysis of SMLM datasets. An overview of all clustering methods can be found in Ref. 23. davg is defined as the average of the minimum distance between two points. 

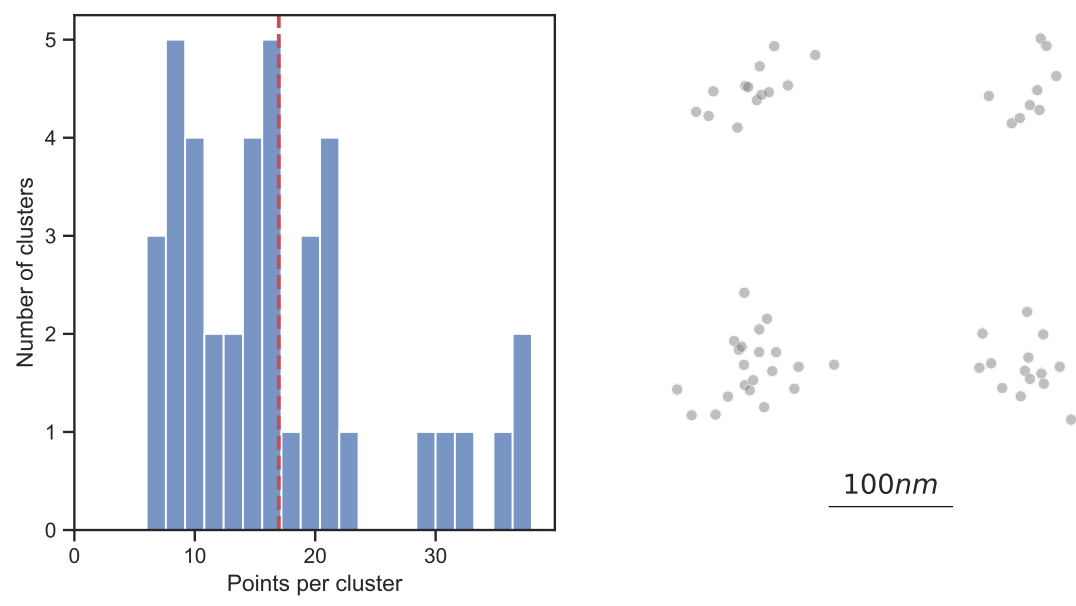

(a) Statistics and samples of 40 clusters extracted from SRM of a neuronal dendrite 10 . Largest cluster diameter: $150 \mathrm{~nm}$. Mean number of localizations per cluster: 17 (red dashed line).
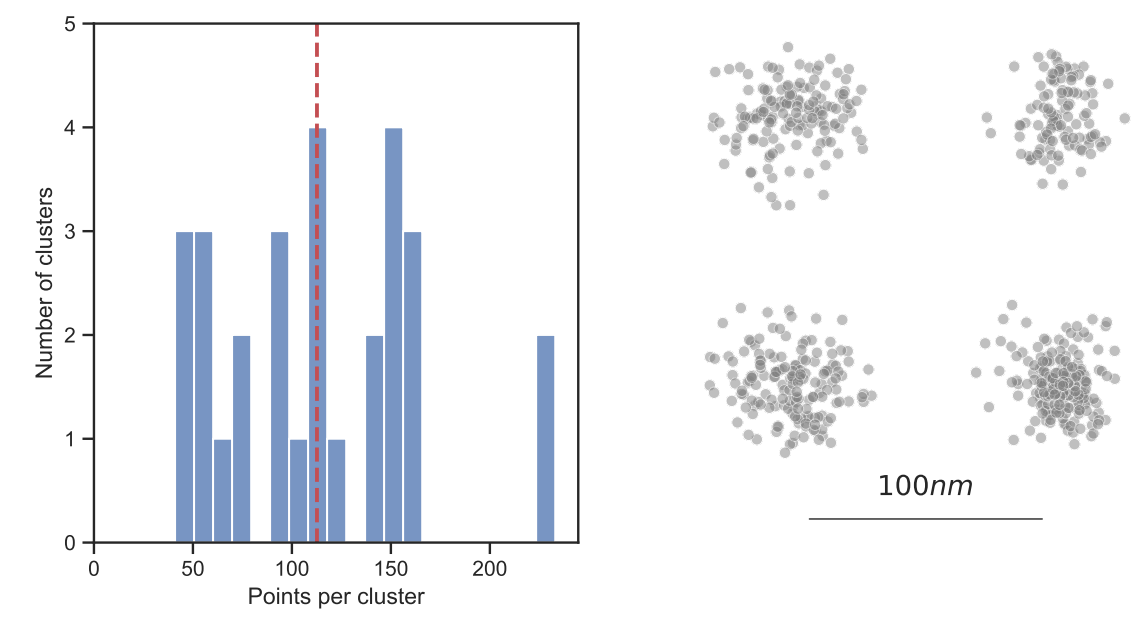

(b) Statistics and samples of 29 Clusters extracted from a DNA Origami trimer dataset. Largest cluster diameter: $77 \mathrm{~nm}$. Mean number of localizations per cluster: 113 (red dashed line).

Figure S1: Cluster libraries: Size distribution (left) and four samples (right) of two libraries of clusters used in this work. 
Step 1

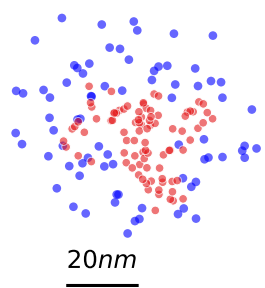

Step 2

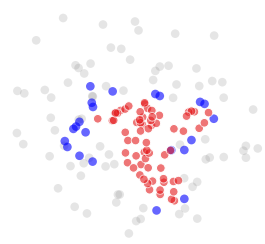

Step 3

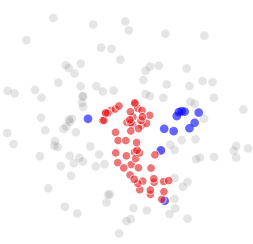

Step 4

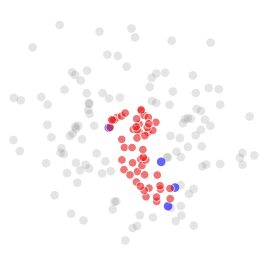

Figure S2: Example of the first four iterative steps of noise removal in DBSCAN (noise free). Points that have the minimum number of neighboring points minPts $=10$ within a given distance $\varepsilon=7 \mathrm{~nm}$ are considered as core points (shown in red). Iteratively, all points which do not satisfy this condition are removed (shown in blue), therefore restricting the number of spurious inclusions. Points that have been excluded in previous iterations are shown in grey. 

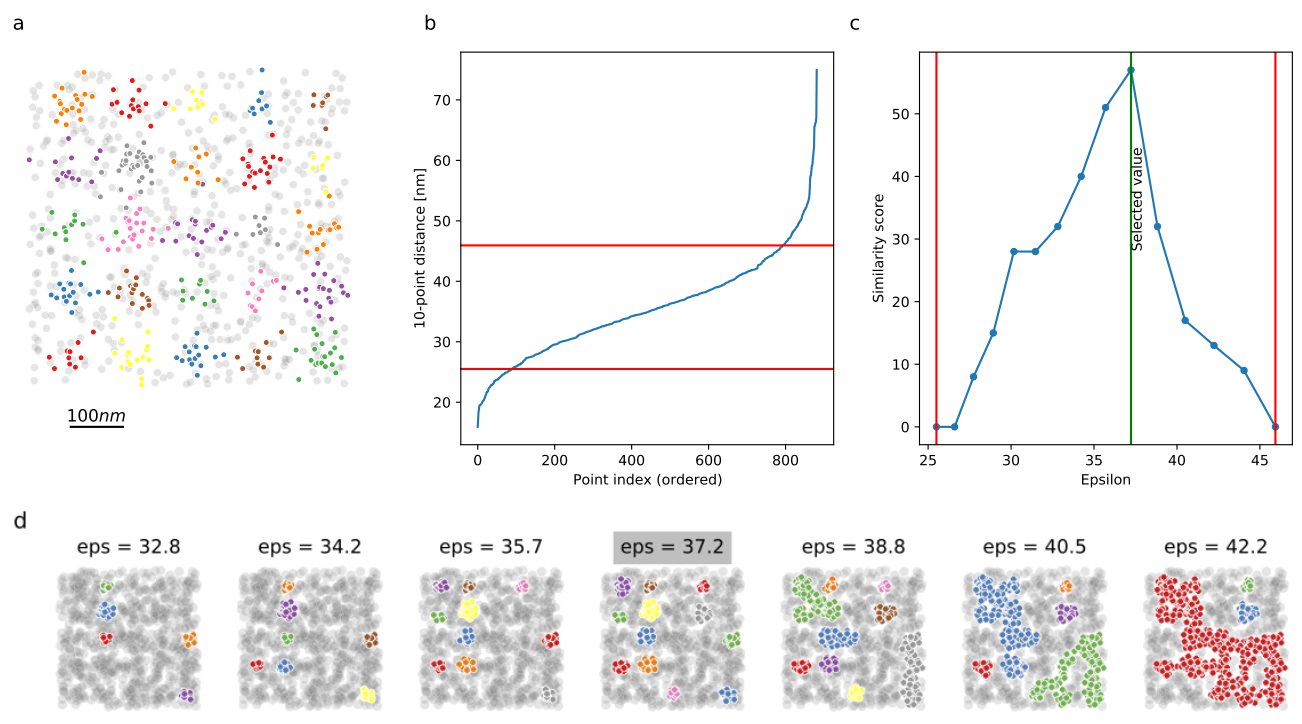

(a) Similarity-based hyperparameter finding with DBSCAN (noisefree)

a

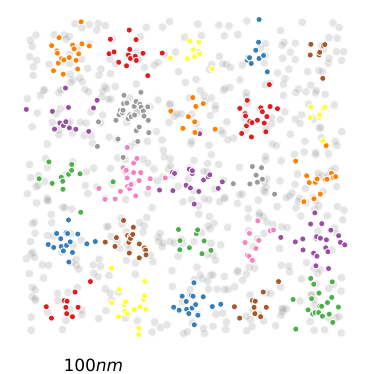

d b

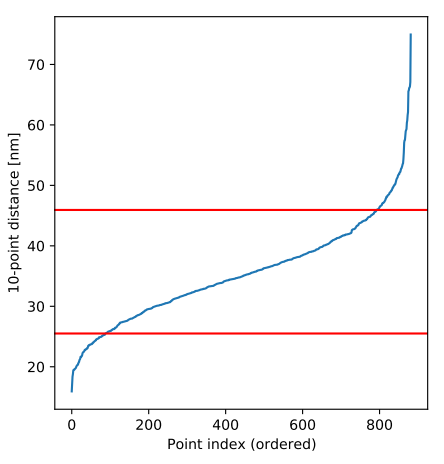

c

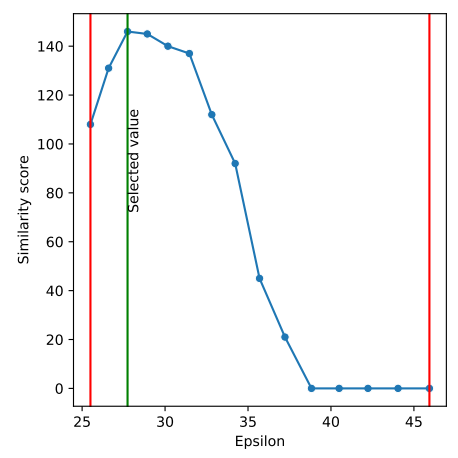

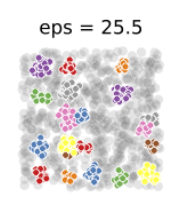
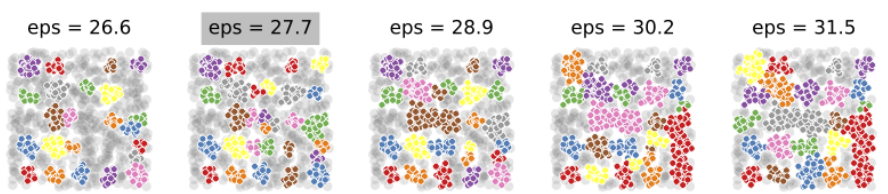

(b) Similarity-based hyperparameter finding with DBSCAN

Figure S3: FINDER 1D applied to configuration shown in Fig. 4 a of the main manuscript. (a) Ground-truth clusters are shown in color, and random noise localizations in grey. (b) 10-th nearest neighbor for all points of the configuration shown in (a), ordered by distance. Red lines indicate the 10th and the 90th quantile of the distribution, which are subsequently used as left and right boundaries of the interval in which in the parameter $\varepsilon$ is searched. (c) A given number of epsilon-values (here 15), are distributed logarithmically within the interval, and their respective clusterings are computed using DBSCAN (noisefree). The plot shows similarity scores within the assembly of clusterings, and the maximal value is chosen as an optimal hyperparameter. (d) Clustering results for the optimal value of epsilon (grey background), and the three lower and higher values within the interval of consideration are shown. See Fig. S19 for an analysis of the variation of the number of $\varepsilon$-values within the interval of interest. 


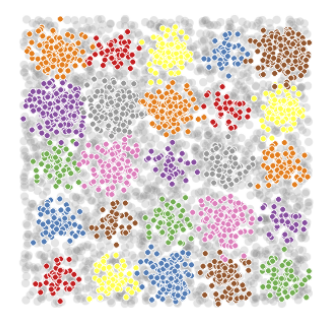

$\underline{100 \mathrm{~nm}}$

d

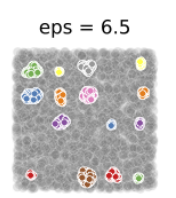

b
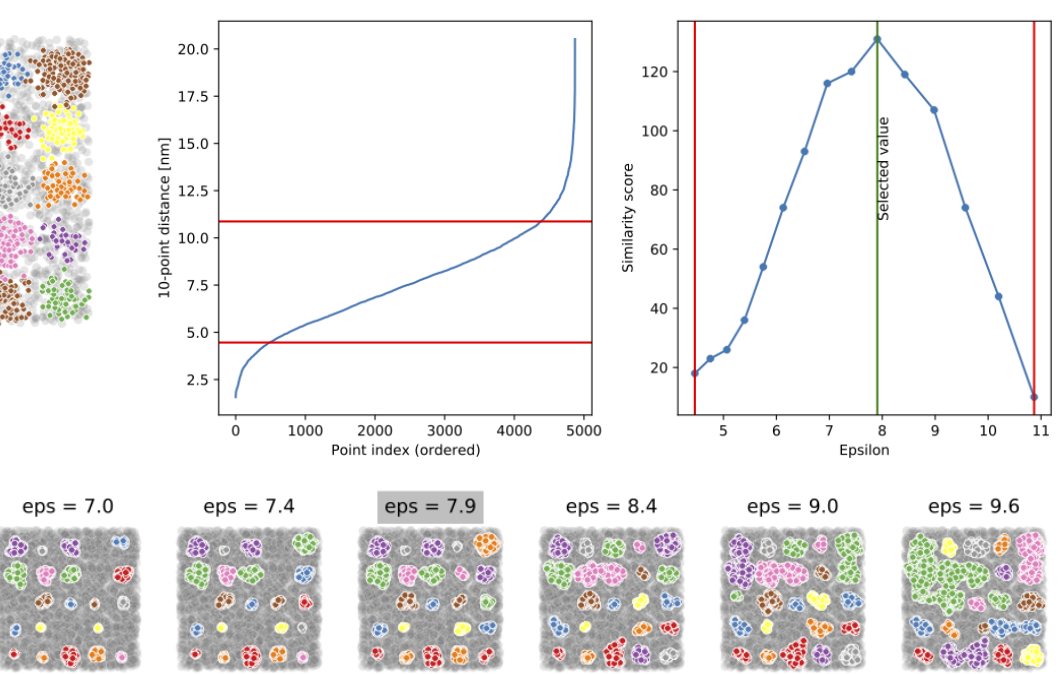

eps $=9.6$

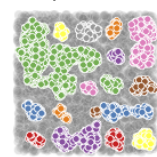

(a) Similarity-based hyperparameter finding with DBSCAN (noisefree)

a

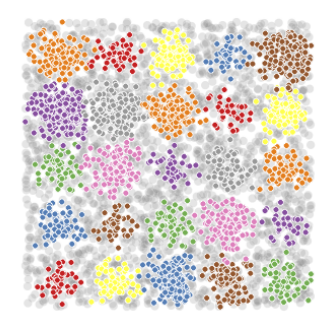

$100 \mathrm{~nm}$

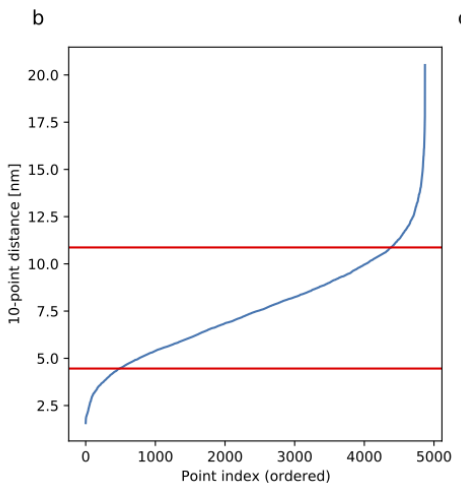

c
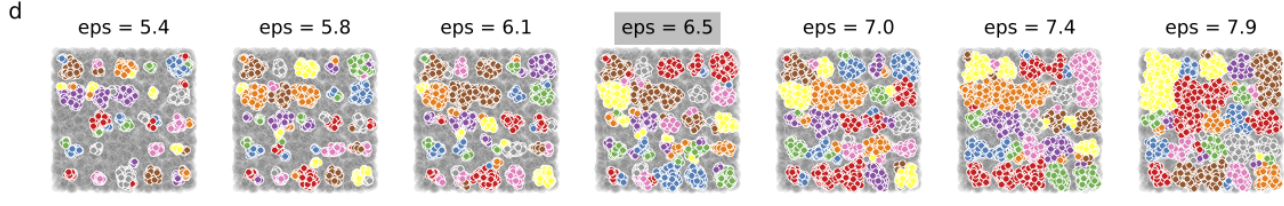

(b) Similarity-based hyperparameter finding with DBSCAN

Figure S4: FINDER 1D applied to configuration shown in Fig. 4 d of the main manuscript. For details, see caption of Fig. S3. See Fig. S19 for an analysis of the variation of the number of $\varepsilon$-values within the interval of interest. 


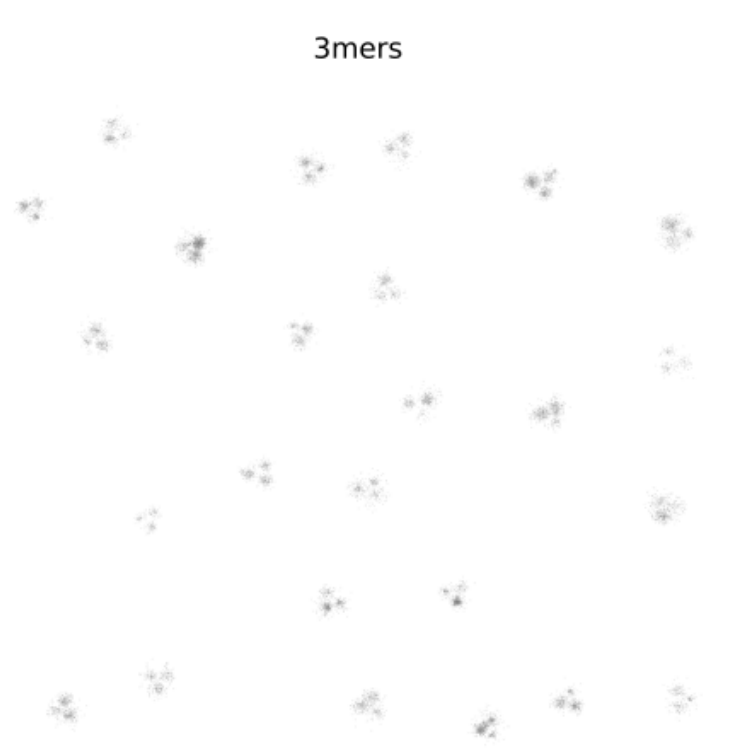

$500 \mathrm{~nm}$

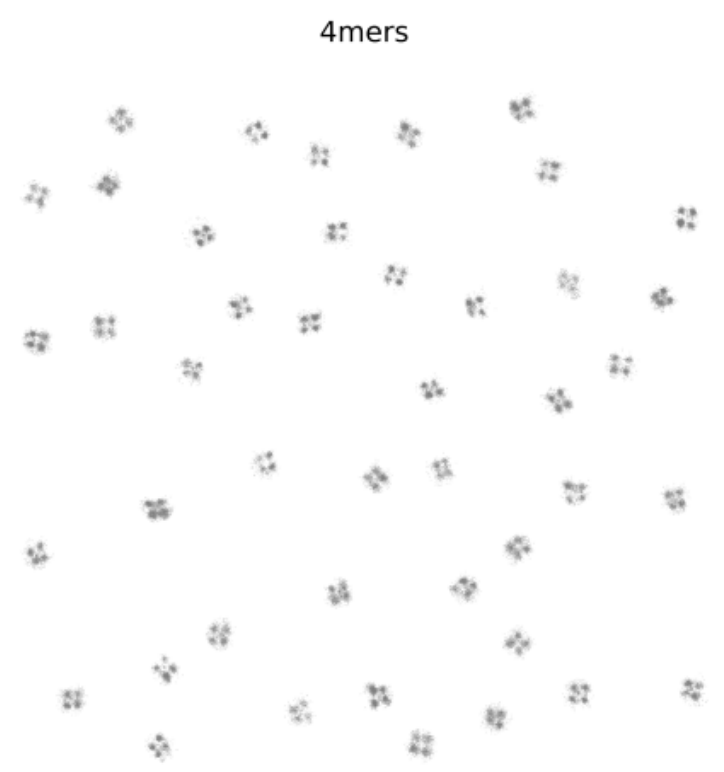

$\underline{500 \mathrm{~nm}}$
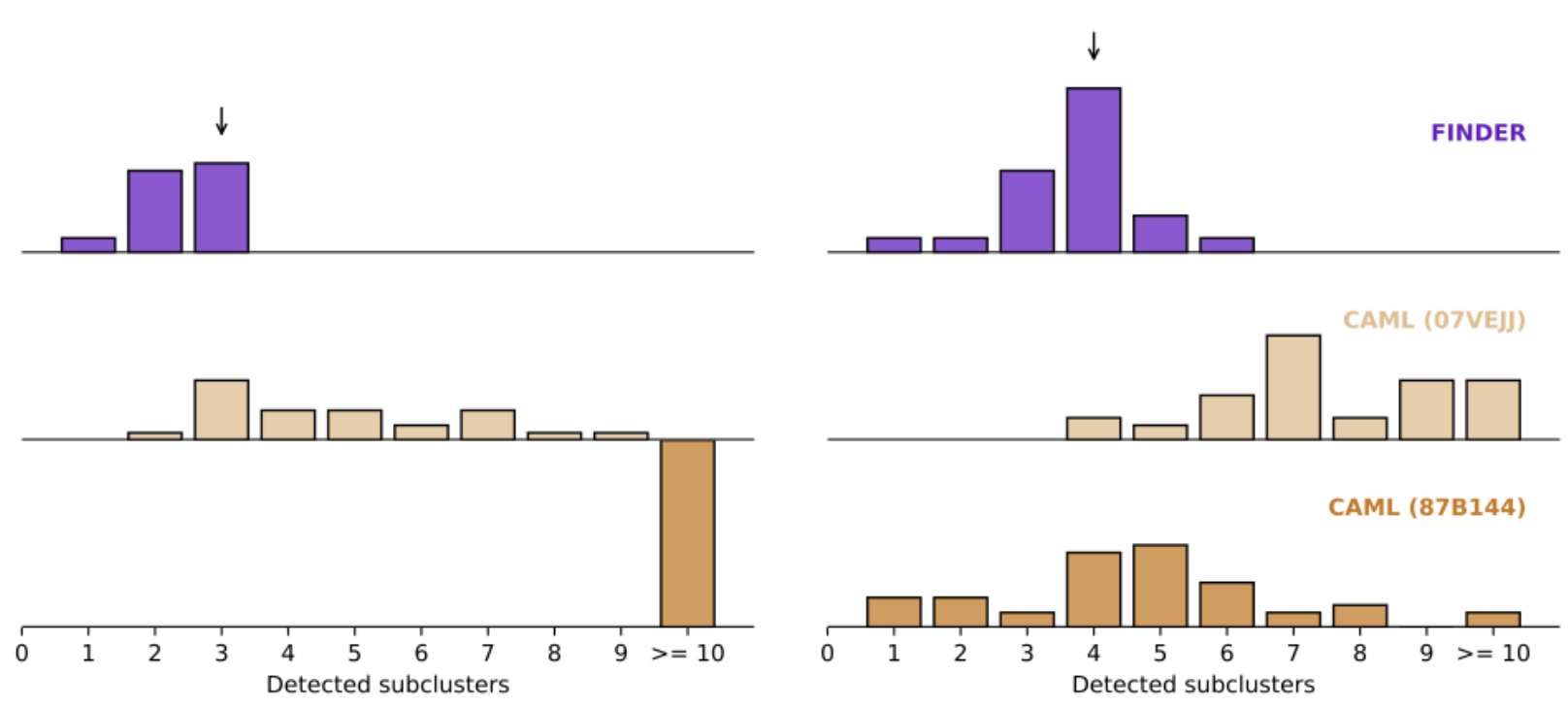

Figure S5: Relates to Fig. 3. Same computation as in Fig. 3, but without added random noise. Here, the optimal radial parameters identified by FINDER for DBSCAN (noisefree) are $\varepsilon=8.2$ and $\varepsilon=4.2$ for minPts $=10$ for the trimer and the tetramer dataset, respectively. 3-mers are not recognized by CAML (87B144). 


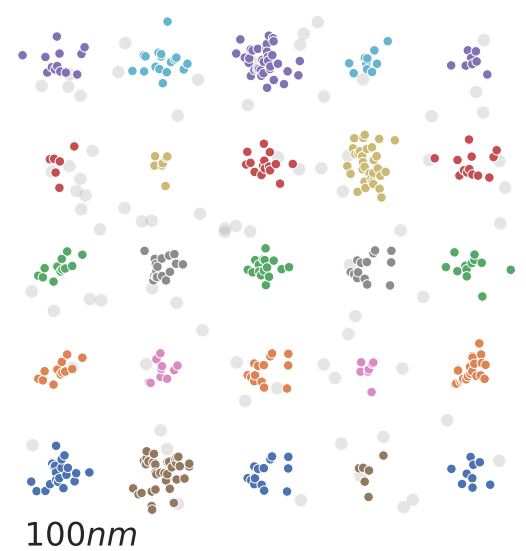

$100 \mathrm{~nm}$

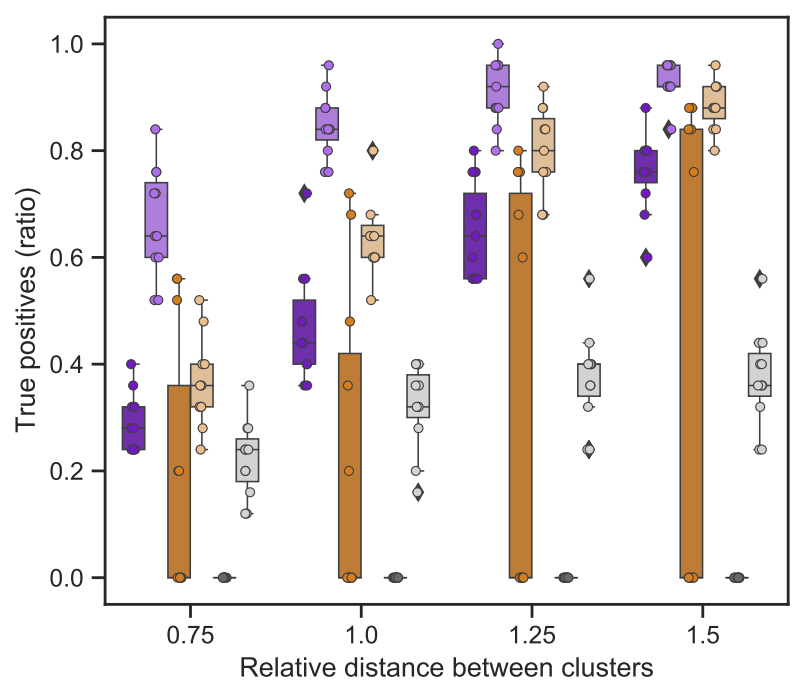

FINDER with DBSCAN (noisefree)

FINDER with DBSCAN

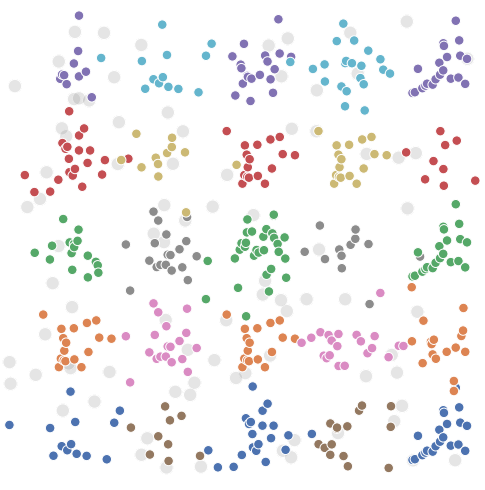

$100 \mathrm{~nm}$

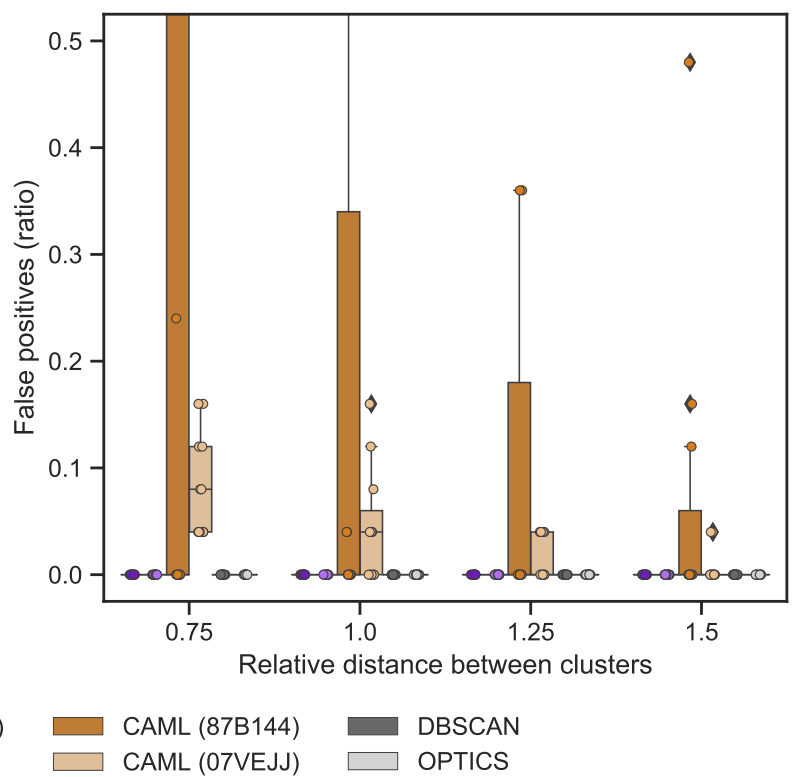

Figure S6: Relates to Fig. 4 a of the main manuscript. Clusters from a SMLM dataset of a synapse [10] (see Fig. 1(a) are randomly assigned to a $5 \times 5$ grid. Based on the number of localizations, a ratio of 0.2 of random noise localizations was then added to the domain. The spacing in between clusters was decreased, here shown as multiples of the maximal cluster diameter of the cluster library, here $150 \mathrm{~nm}$. Top row: Two samples with highest and lowest spacing. Boxplots show the number of true and false positive cluster detections. Parameters used for DBSCAN [18] were $\varepsilon=10 \mathrm{~nm}$, minPts $=10$ and for OPTICS 21]: minPts $=20, \mathrm{xi}=0.05$, max epsilon $=100 \mathrm{~nm}$. 

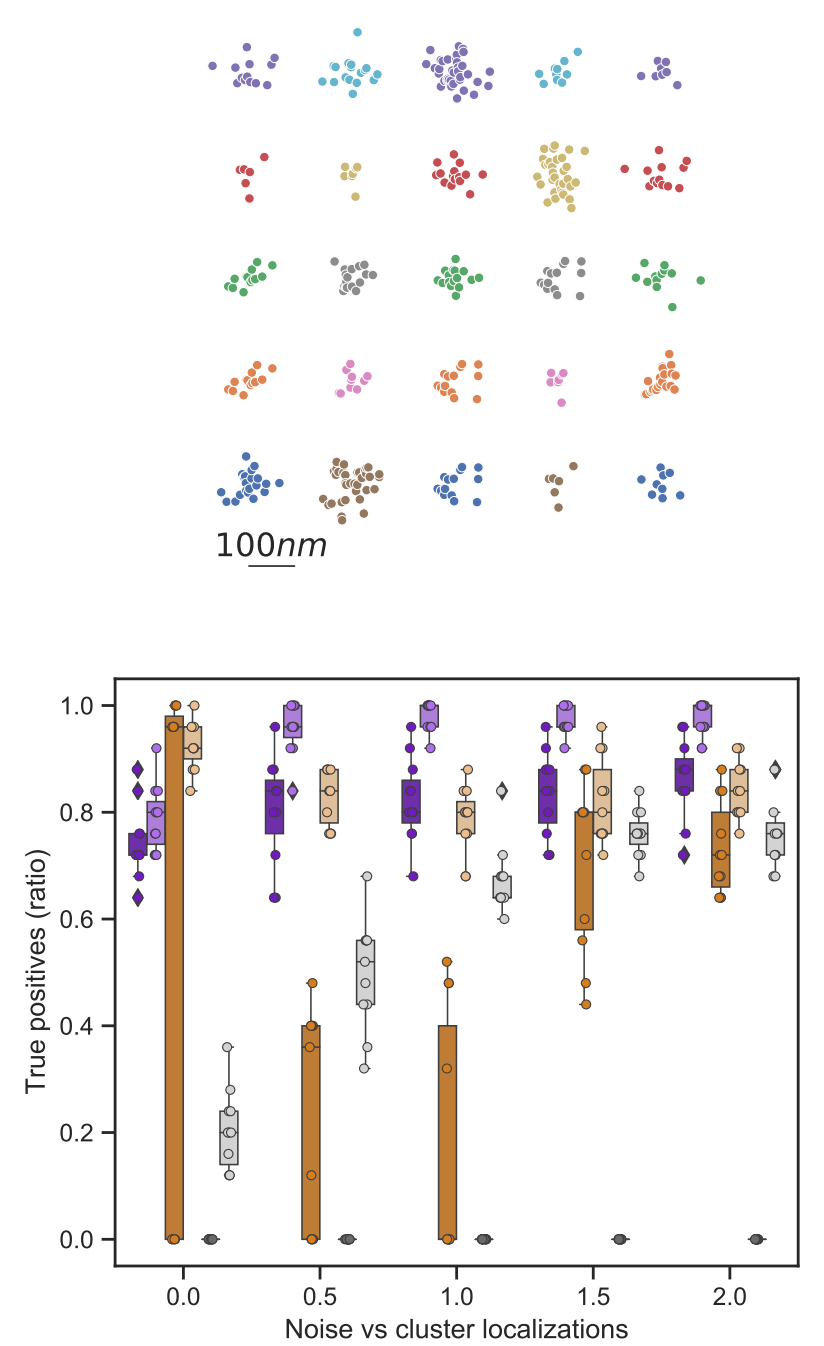

FINDER with DBSCAN (noisefree) $\square$ FINDER with DBSCAN
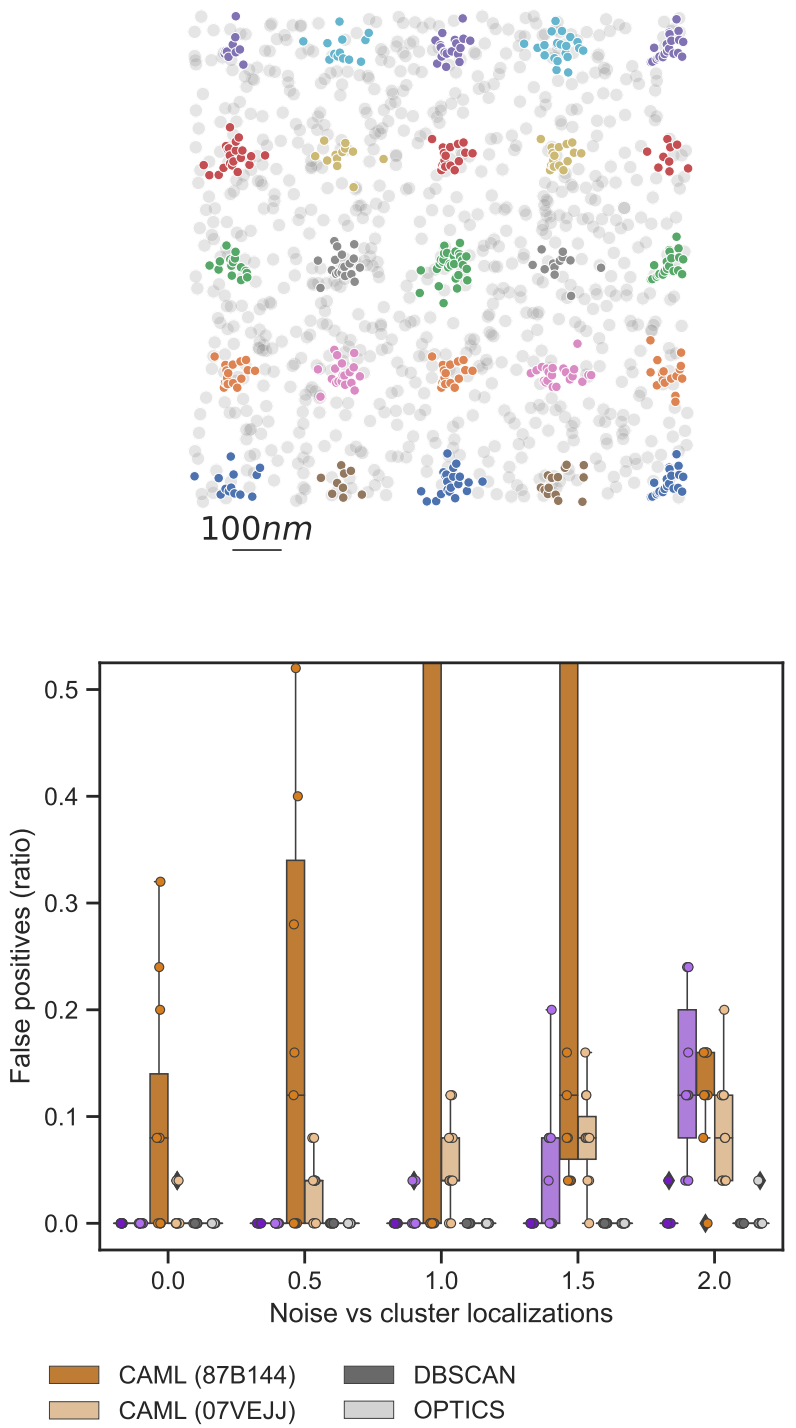

Figure S7: Relates to Fig. 4 b of the main manuscript. Clusters from a SMLM dataset of a synapse 10 (see Fig. 1(a) are randomly assigned to a $5 \times 5$ grid, with spacing $225 \mu \mathrm{m}$. Based on the number of localizations, an increasing ratio of random noise localizations was then added to the domain. See caption of Fig. S6 for details. 

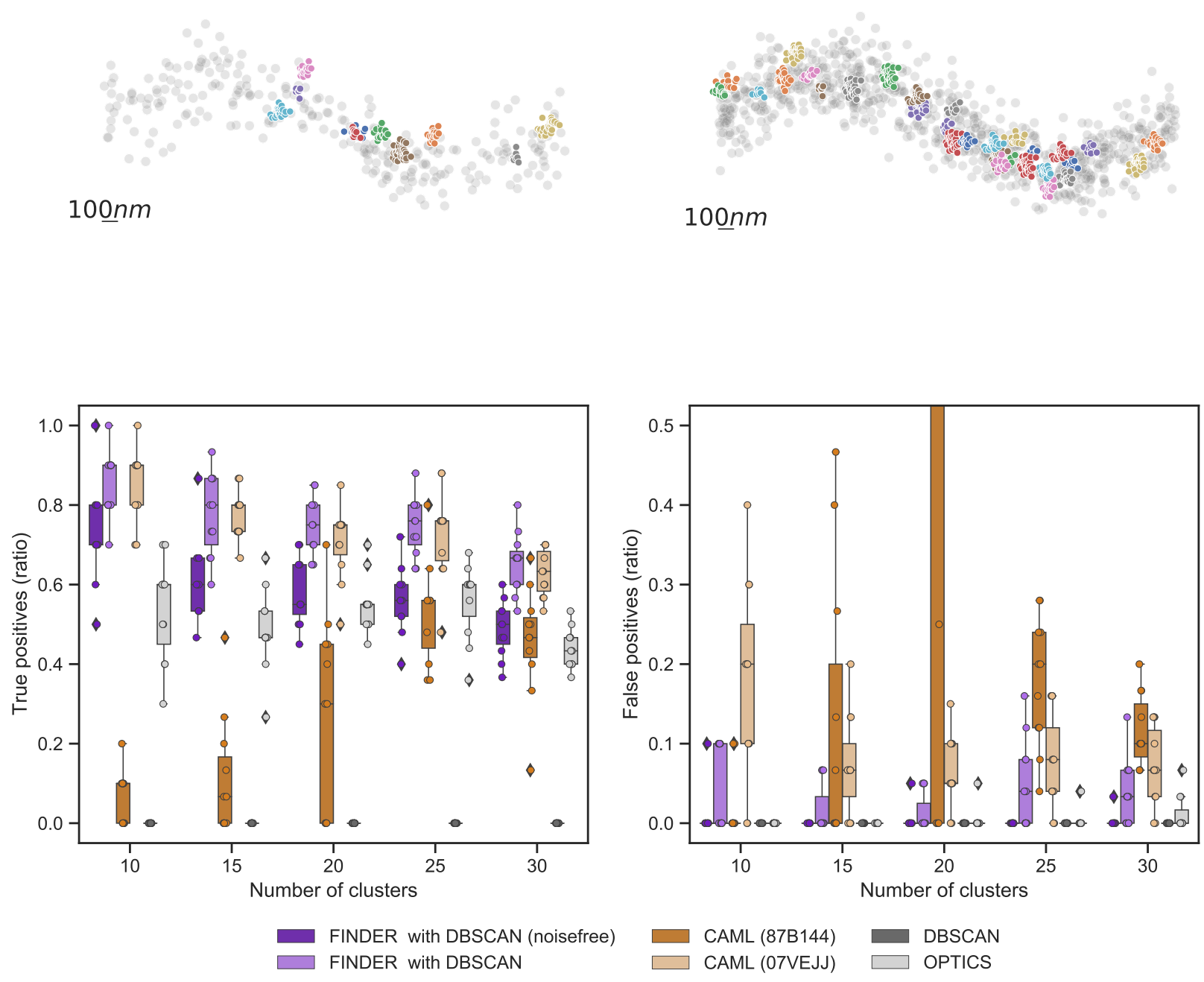

Figure S8: Relates to Fig. 4 c of the main manuscript. Clusters from a SMLM dataset of a synapse [10] (see Fig. 1(a) are randomly assigned to a sinusoidal path. Based on the number of localizations of the added clusters, a ratio of 1.5 of random noise localizations was then added to the domain. The number of clusters added to the domain was then increased from 10 to 30, therefore increasing noise and overlap. See caption of Fig. $\mathrm{S6}$ for details. 


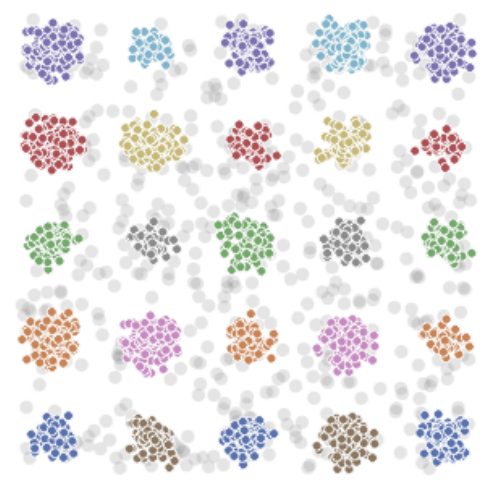

$100 \mathrm{~nm}$

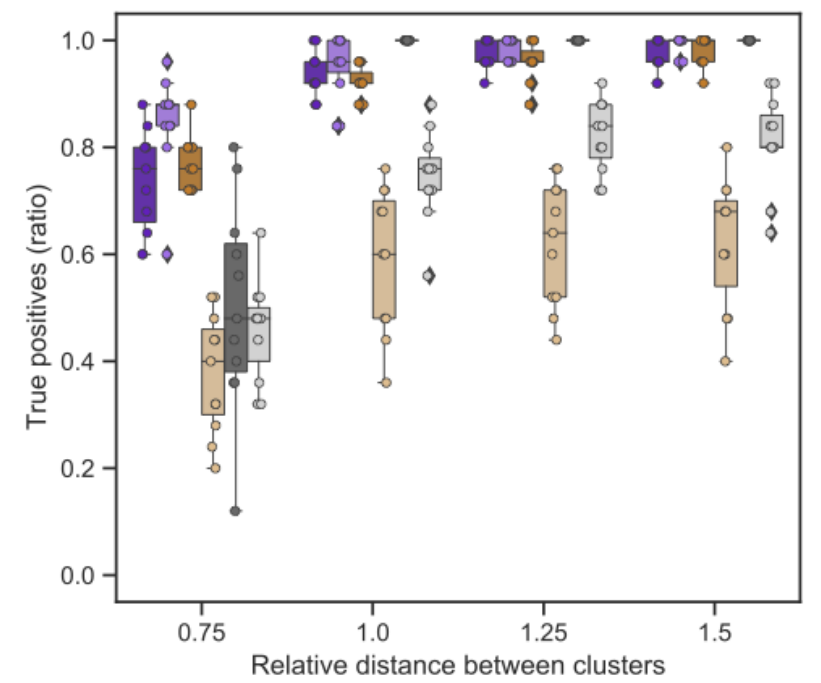

FINDER with DBSCAN (noisefree)

FINDER with DBSCAN

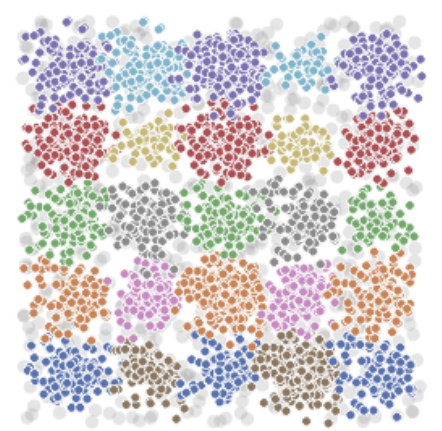

$100 \mathrm{~nm}$

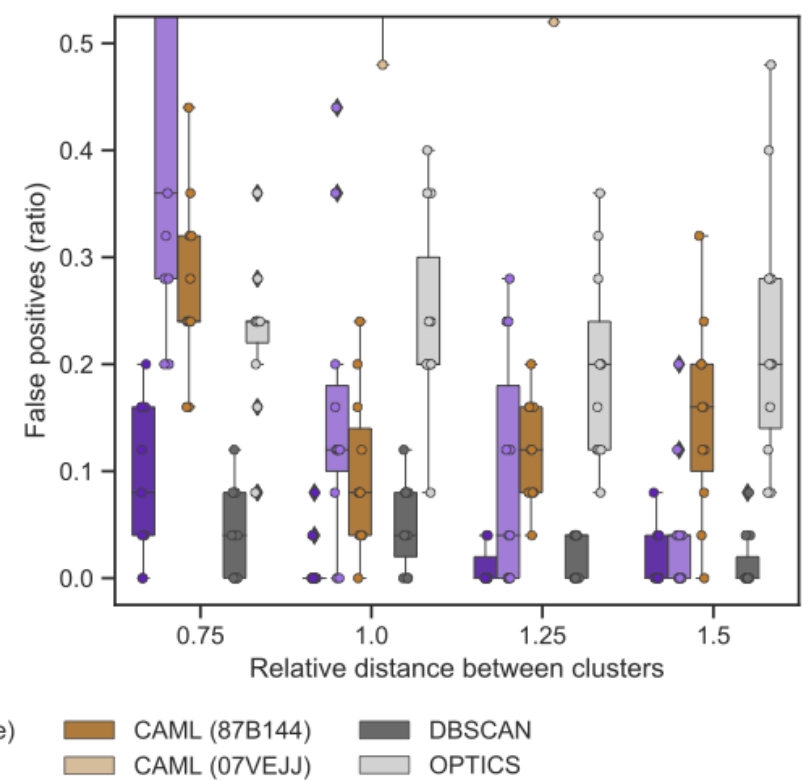

Figure S9: Relates to Fig. 4 d of the main manuscript. Clusters from a DNA origami dataset (see Fig. 1(b) are randomly assigned to a $5 \times 5$ grid, with spacing $115 \mu \mathrm{m}$. Based on the number of localizations, an increasing ratio of random noise localizations was then added to the domain. See caption of Fig. S6 for details. 


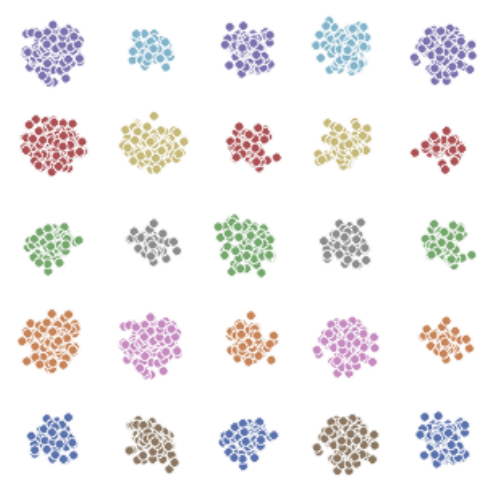

$100 \mathrm{~nm}$

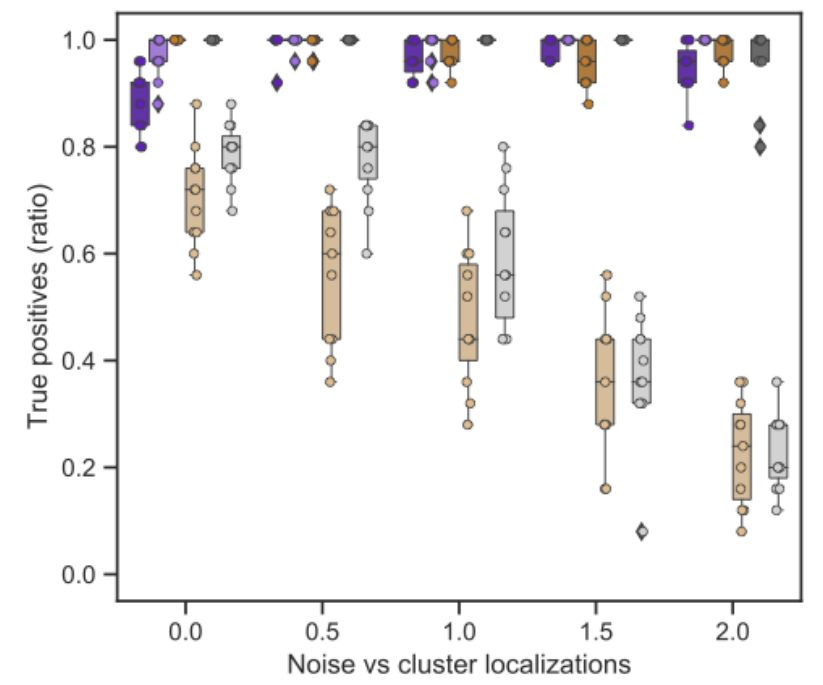

FINDER with DBSCAN (noisefree) FINDER with DBSCAN

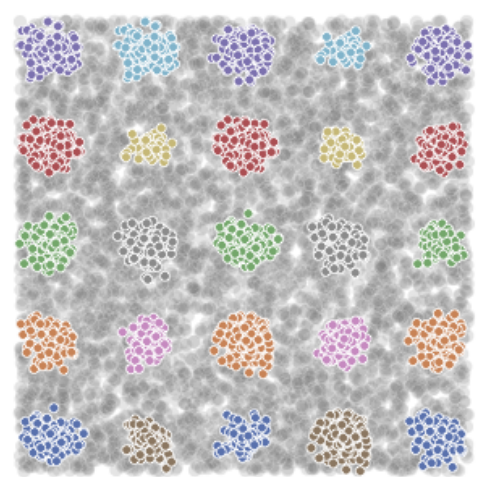

$100 \mathrm{~nm}$

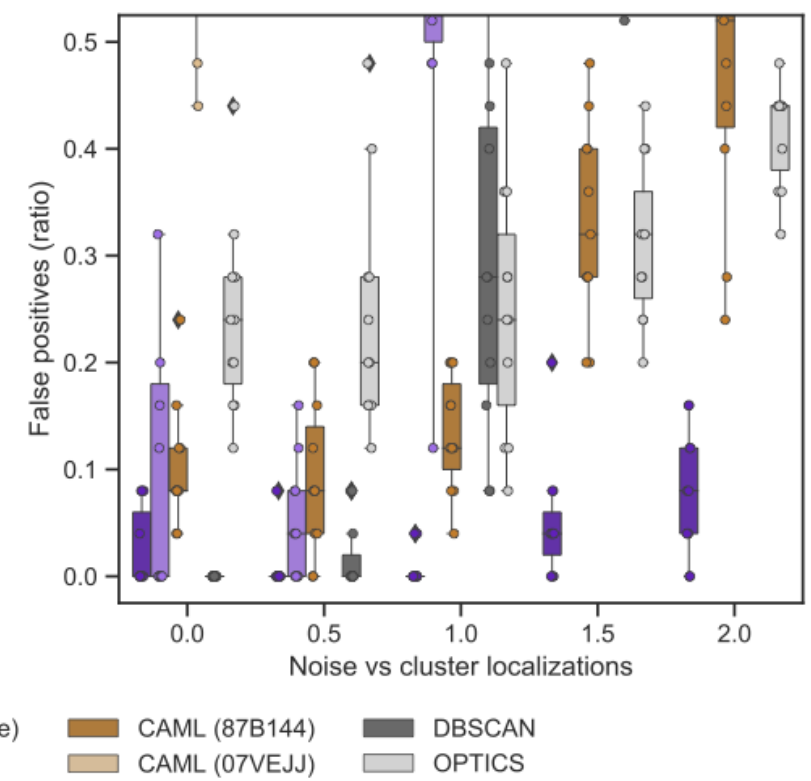

Figure S10: Relates to Fig. 4 e of the main manuscript. Clusters from a DNA origami dataset (see Fig. 1(b)] are randomly assigned to a $5 \times 5$ grid. Based on the number of localizations, a ratio of 0.2 of random noise localizations was then added to the domain. The spacing in between clusters was decreased, here shown as multiples of the maximal cluster diameter of the cluster library, here $77 \mathrm{~nm}$. See caption of Fig. S6 for details. 


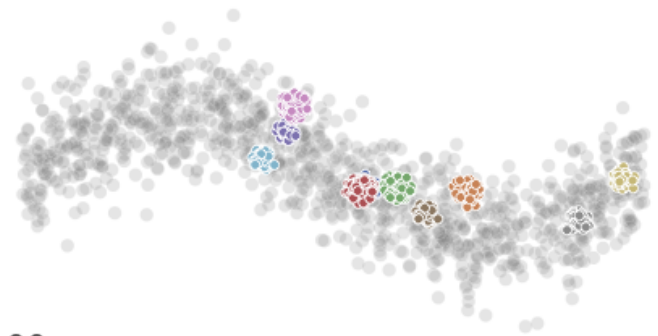

$100 \mathrm{~nm}$

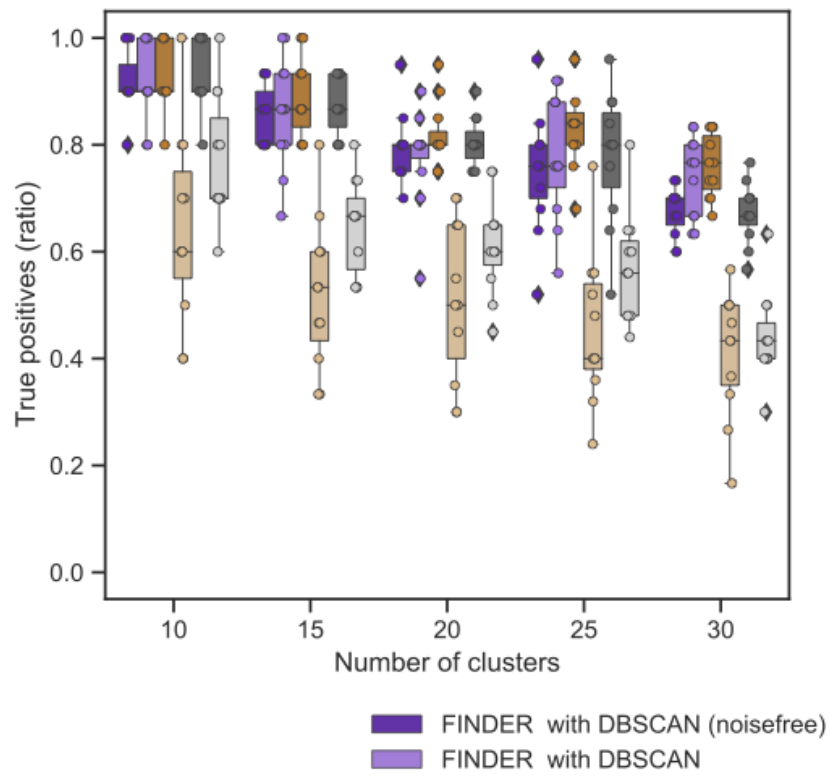

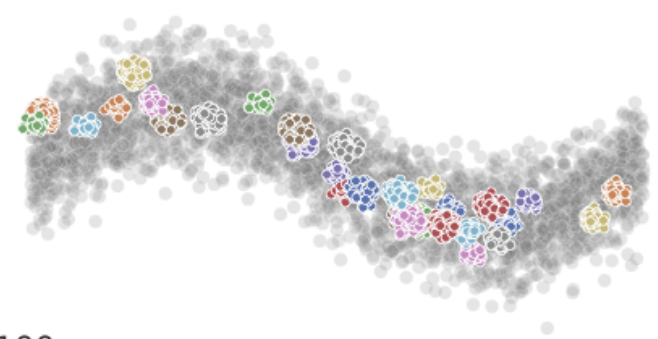

$100 \mathrm{~nm}$

Figure S11: Relates to Fig. 4 f of the main manuscript. Clusters from a DNA origami dataset (see Fig. 1(b)] are randomly assigned to a sinusoidal path. Based on the number of localizations of the added clusters, a ratio of 1.0 of random noise localizations was then added to the domain. The number of clusters added to the domain was then increased from 10 to 30, therefore increasing noise and overlap. See caption of Fig. S6 for details. 

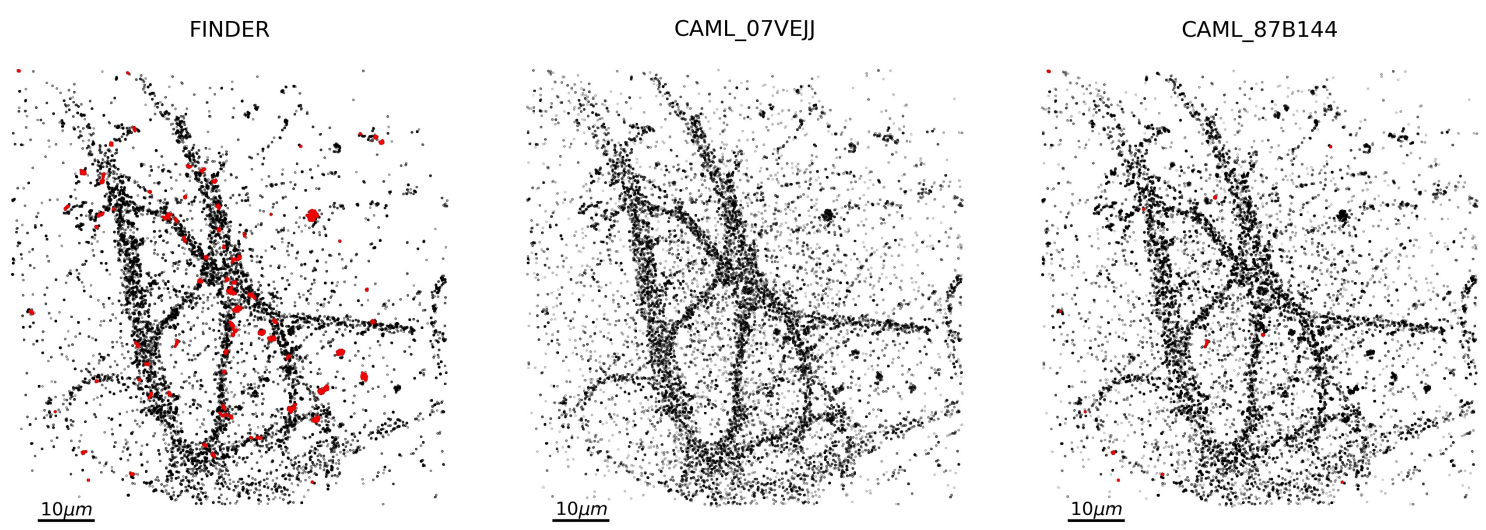

Figure S12: Related to Fig. 5 All localizations not identified as noise. Localizations assigned to clusters of more than 400 are highlighted in red. 


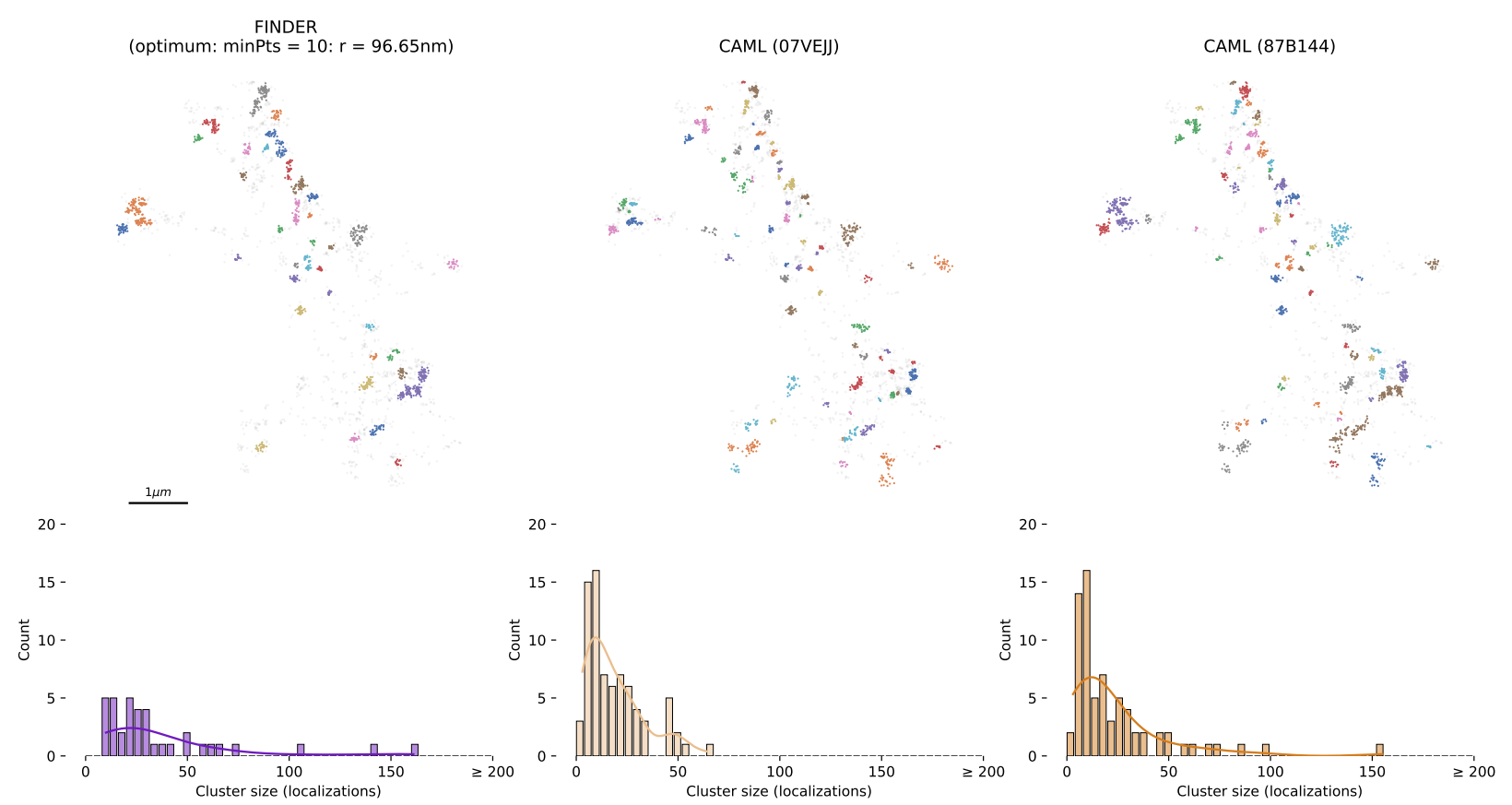

(a) The top row depicts selected clusters (colored points) and localizations that were classified as noise (grey points with transparency). The bottom row shows the distribution of the size of identified clusters.
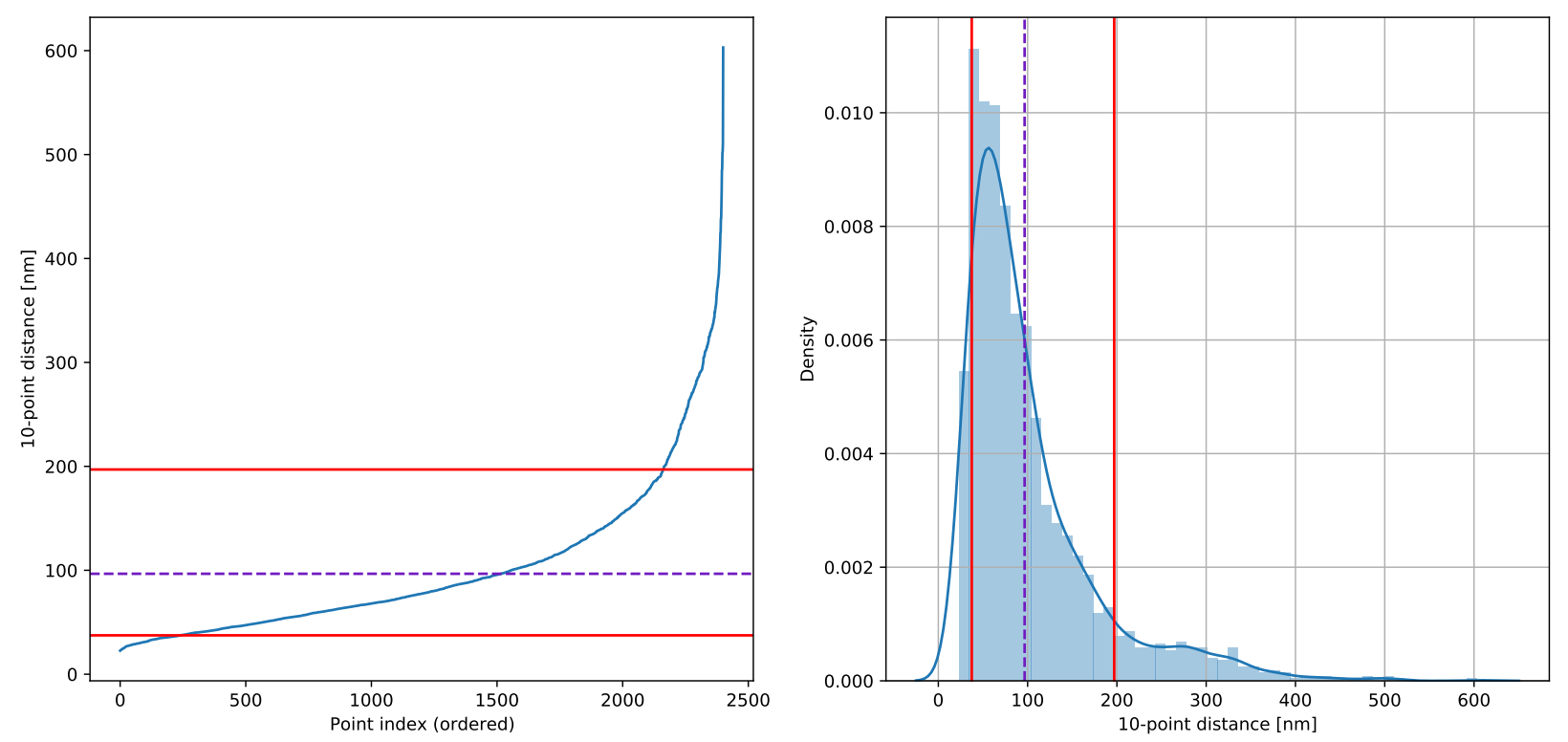

(b) Statistics for the 10th-neighbor distances. Left: Distances for all points, ordered by length. Right: Distribution of distances. The red lines are the 10th and the 90th percentile of the distribution. The violet dashed line denotes the value for $\epsilon$ identified by FINDER.

Figure S13: Analysis analogous to Fig. 5 , but for super resolved neuronal AMPA receptor localizations [10. As in Fig. 5, FINDER identifies fewer clusters with few localizations (cluster size < 25). See Fig. S18 for an analysis of the full phasespace. 

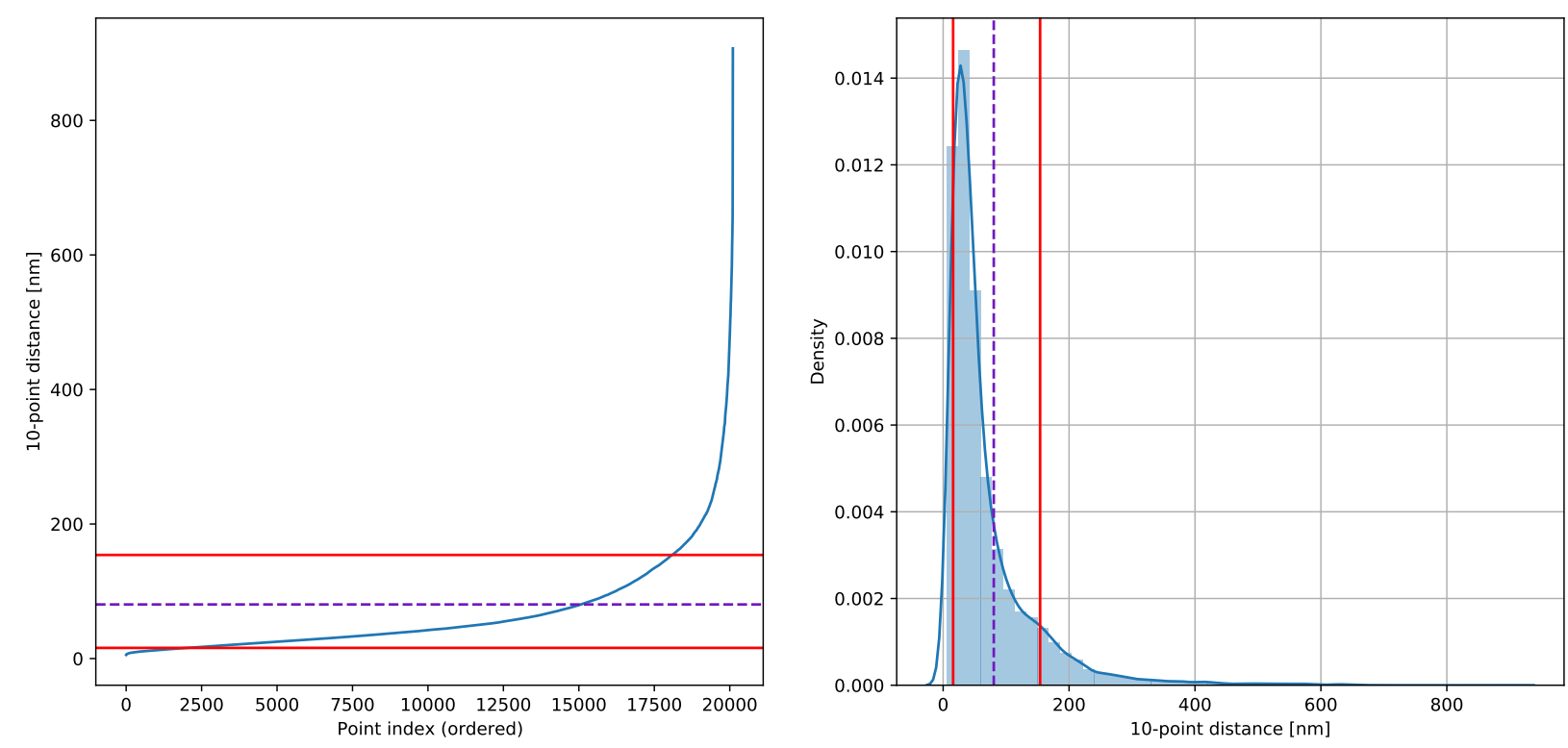

Figure S14: Statistics for the 10th-neighbor distances for the case analyzed in Fig. 5. Left: Distances for all points, ordered by length. Right: Distribution of distances. The red lines are the 10th and the 90th percentile of the distribution. The violet dashed line denotes the optimal value for $\epsilon$ identified by FINDER.
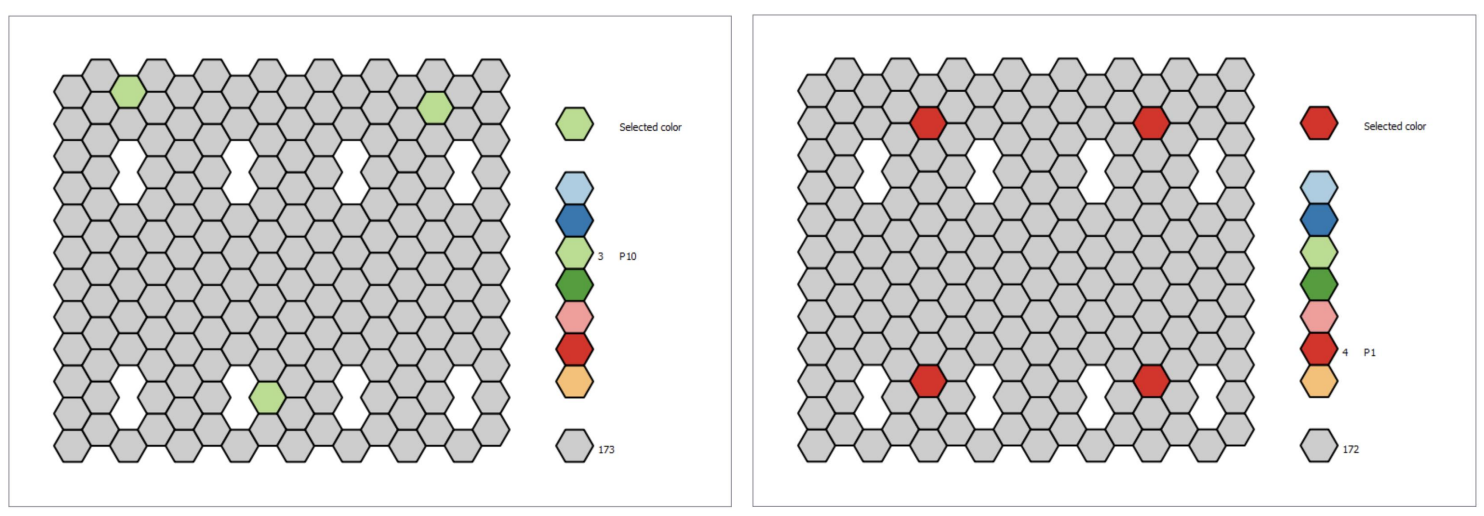

Figure S15: Left: 3-fold symmetric DNA origami containing 3 binding sites. Right: 4-fold symmetric DNA origami containing 4 binding sites. 


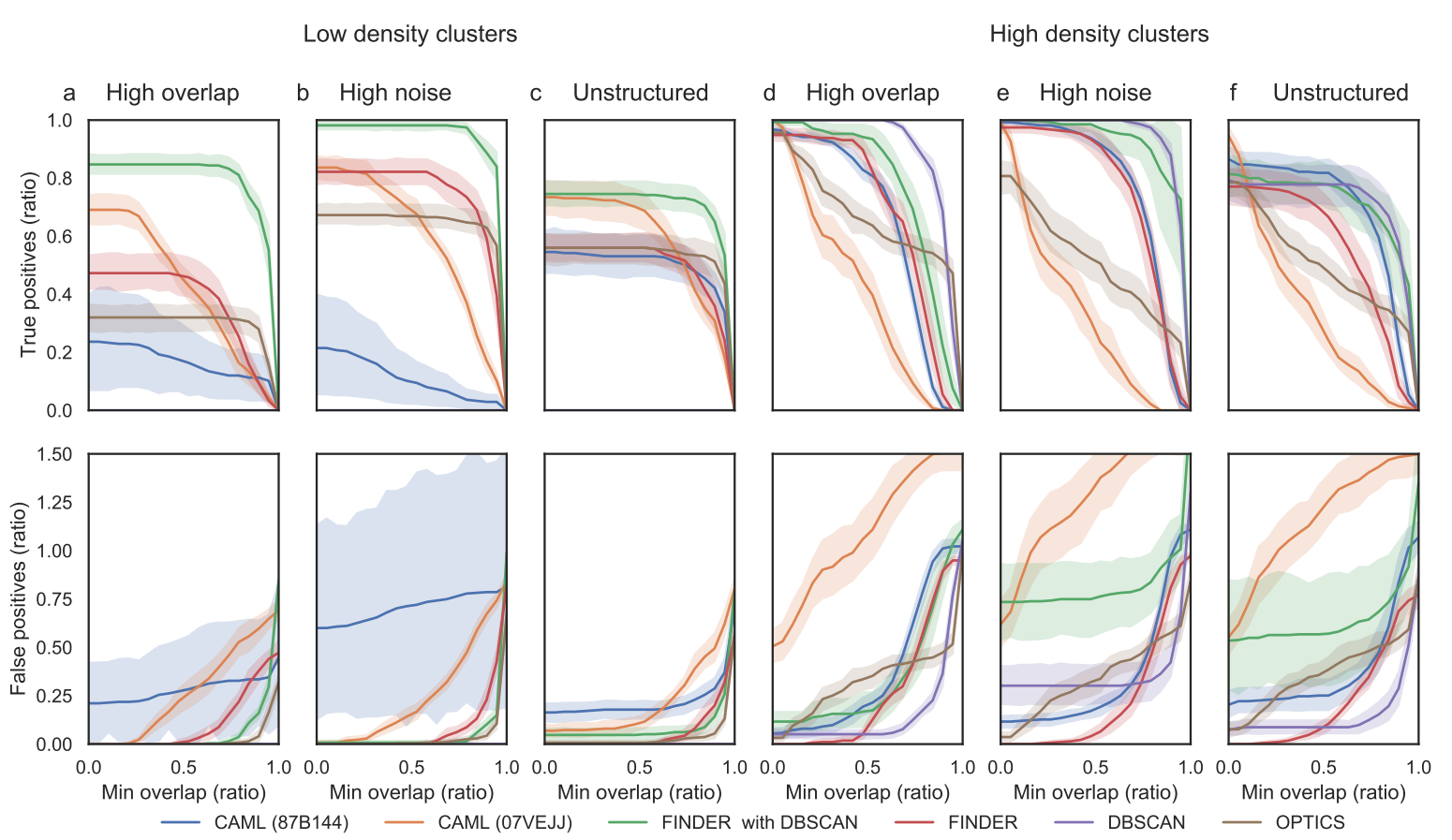

Figure S16: Re-evaluation of the results from Fig. 4. When comparing the clustering outcome to the ground truth, the required overlap between the detected and the ground truth cluster is varied here from 0 to $1(100 \%)$. Note that the clustering outcome does not change with the variation of this threshold - only the ratio of clusters that are attributed to being true or false positives changes. Ratios are given with respect to the number of ground truth clusters. 

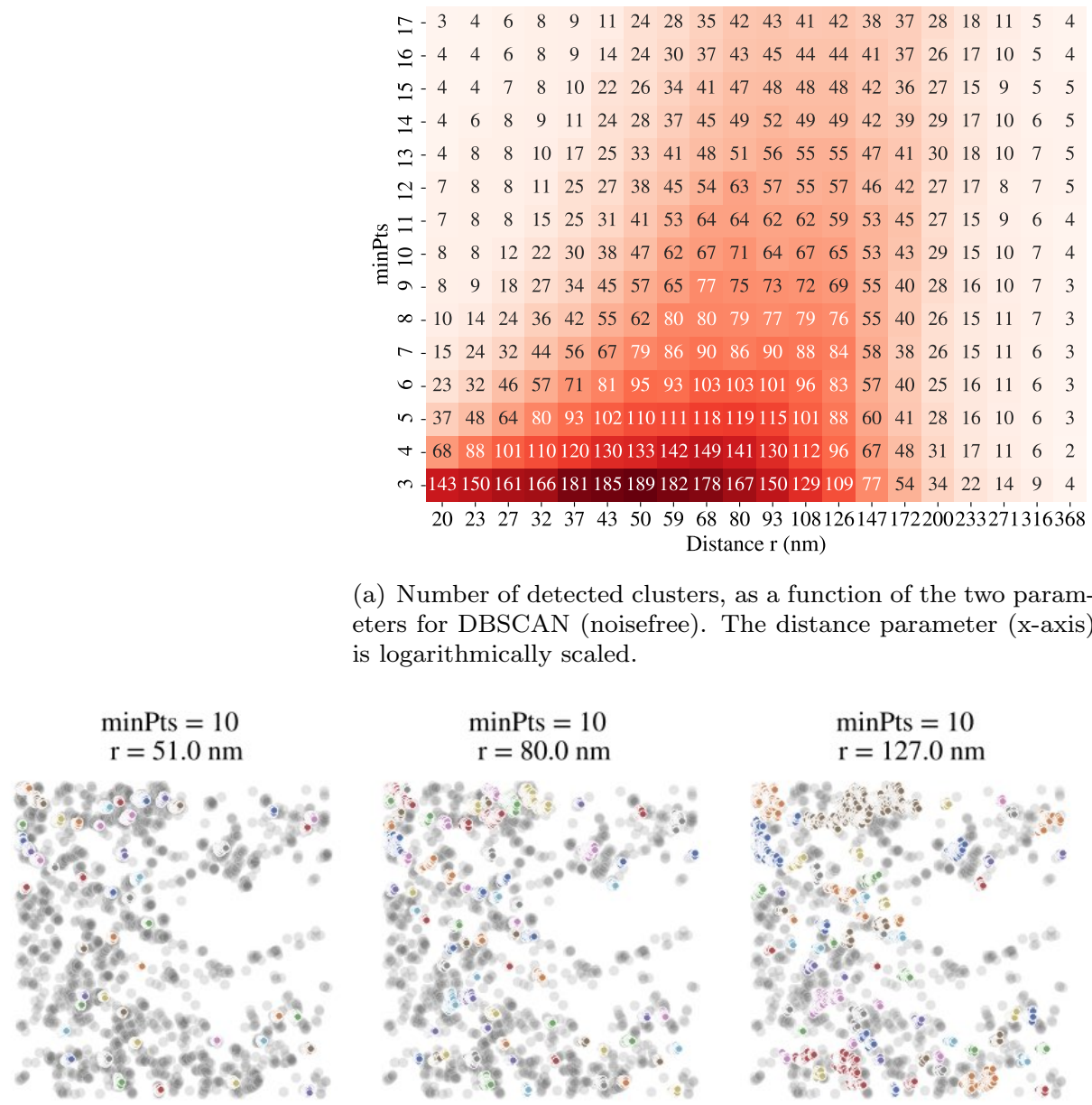

(a) Number of detected clusters, as a function of the two parameters for DBSCAN (noisefree). The distance parameter (x-axis) is logarithmically scaled.
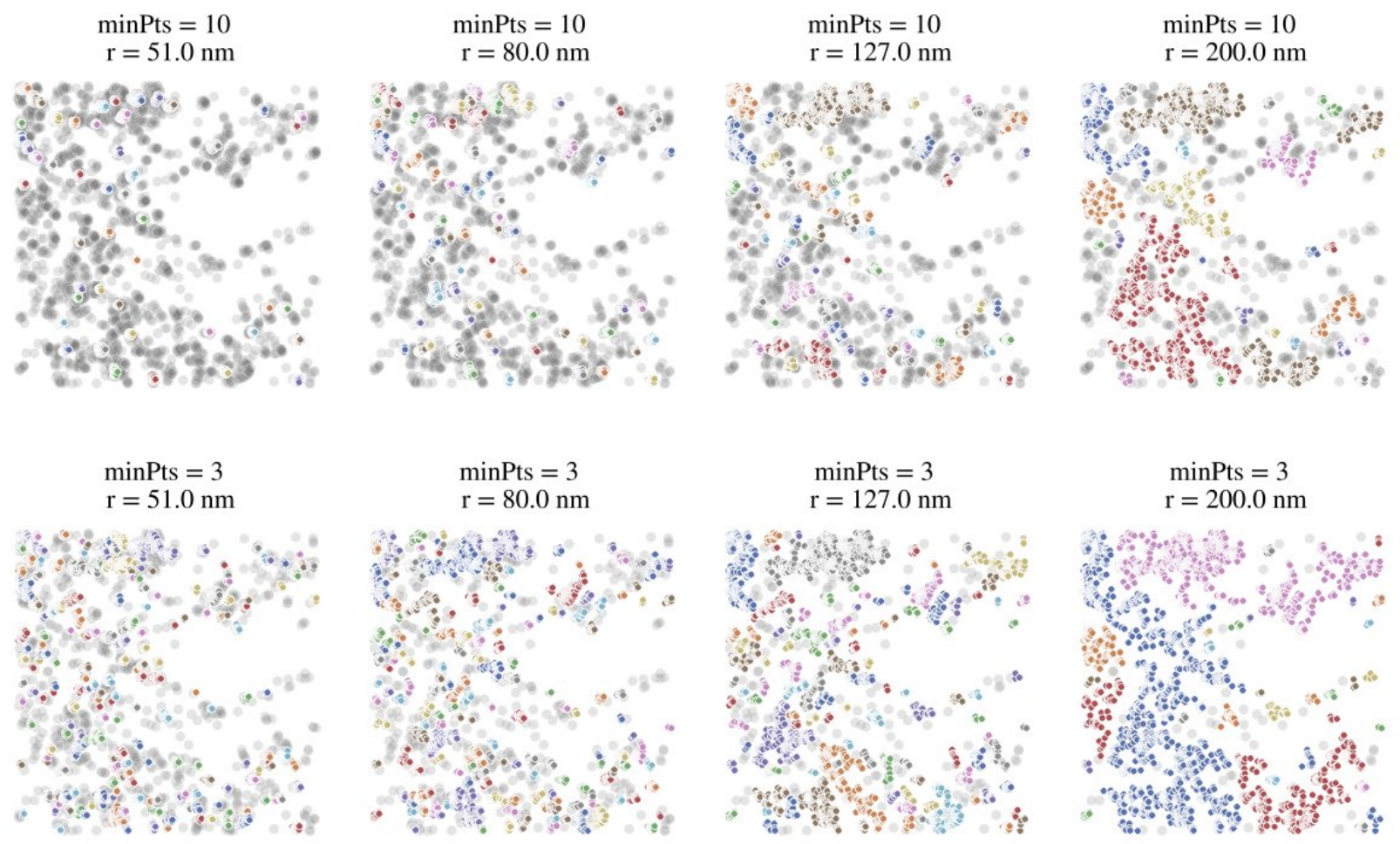

(b) Examples of clustering outcomes for minPts $=10$ (top row) and $\operatorname{minPts}=3$ (bottom row).

Figure S17: Exploration of clustering outcomes with DBSCAN (noisefree) for the localizations within the red rectangle in Fig. 5. Increasing minPts and decreasing $\varepsilon$ have the effect of excluding less dense - or random - cluster formations and breaking up large clusters. For instance in panel (a) at $\varepsilon=200 \mathrm{~nm}$, with minPts $=3,34$ clusters are detected. Increasing minPts first leads to a decrease of the number of detected clusters ( 25 clusters for minPts $=6$ ), as small, less dense clusters are excluded, and then an increase (30 detected clusters for minPts $=30)$, as larger clusters are broken up. Both effects overlap, and repeat, depending on the statistics of the localizations. In order to exclude the spurious clusters detected at low thresholds, the first steep decrease in cluster densities is excluded by setting minPts $=10$, see also Fig. S18. 

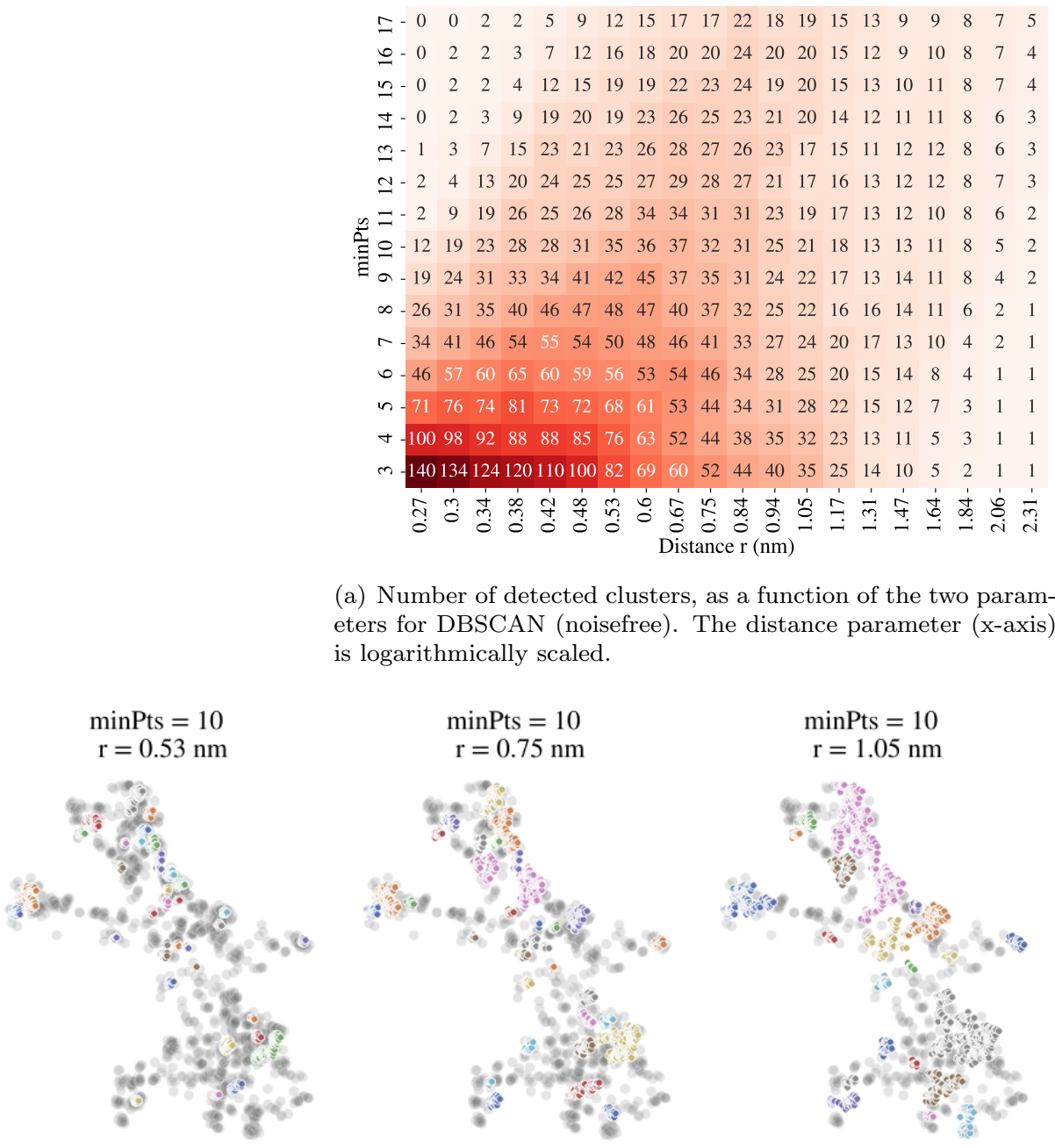

(a) Number of detected clusters, as a function of the two parameters for DBSCAN (noisefree). The distance parameter (x-axis) is logarithmically scaled.
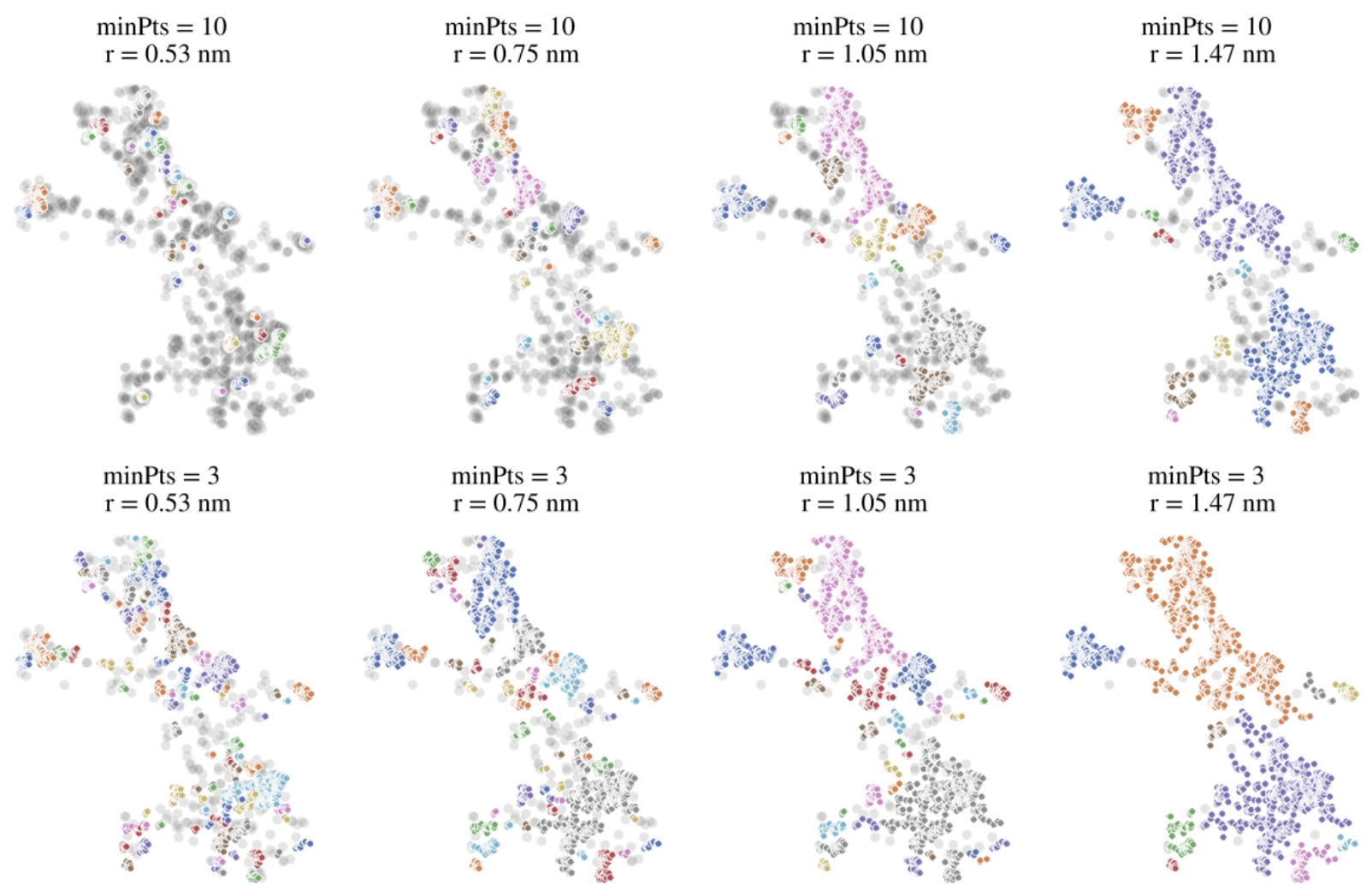

(b) Examples of clustering outcomes for minPts $=10$ (top row) and minPts $=3$ (bottom row).

Figure S18: Exploration of clustering outcomes for the localizations in Fig. 5, analysis analogous to Fig. S17. 

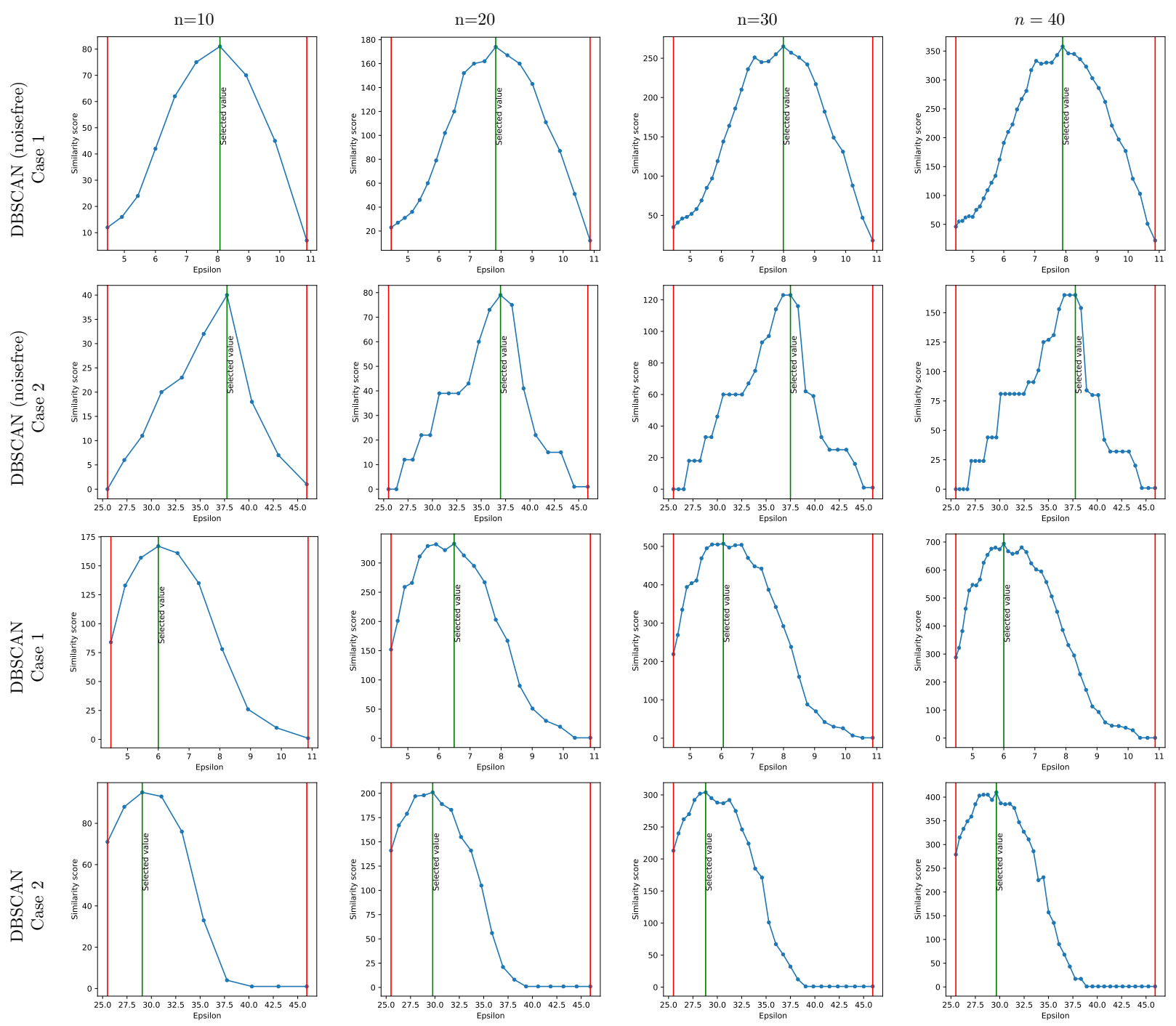

Figure S19: Analysis of examples shown in Figs. S3 and S4, for which the number of $\varepsilon$-values within the domain of interest is varied between 10 and 40. It is seen that the similarity score is robust with respect to changes in the number of points. 